\title{
EMBEDDABILITY FOR THREE-DIMENSIONAL CR-MANIFOLDS
}

\author{
DANIEL M. BURNS AND CHARLES L. EPSTEIN
}

\section{CONTENTS}

0 . Introduction

1. The spectral theory of $\square_{b}$

2. The analyticity properties of families

3. The first and second variations

4. Nonembeddability is generic

5. Small perturbations of the standard sphere

\section{INTRODUCTION}

A question of principal interest in the theory of compact, three-dimensional CR-manifolds is to understand when a given strictly pseudoconvex, CR-structure can be realized by an embedding in $\mathbb{C}^{n}$. We call a structure for which this is possible embeddable, and nonembeddable otherwise.

This question is only of interest in three dimensions as a theorem of Boutet de Monvel states that any strictly pseudoconvex CR-structure, on a compact $(2 d+1)$-manifold, is realizable as an embedding in some $\mathbb{C}^{n}$, provided $d>1$. On the other hand, there is a classical example of Rossi which shows that an arbitrarily small, real analytic, perturbation of the standard structure on the three sphere may fail to be embeddable, [Ros]. Later, Nirenberg constructed $\mathscr{C}^{\infty}$-perturbations for which the associated $\bar{\partial}_{b}$-equation fails to have even local solutions, [N]. In fact, this situation is generic in the $\mathscr{C}^{\infty}$-topology. It is now understood, principally from the work of Eliashberg, that CR-structures with

Received by the editors January 29, 1990 and, in revised form, June 16, 1990; a preliminary version of this paper was presented to the American Mathematical Society at the Ball State regional meeting, October, 1989.

1980 Mathematics Subject Classification (1985 Revision). Primary 53C15, 32G07, 32F30.

Key words and phrases. CR-manifolds, closed range, embeddings.

The first author's research was supported in part by NSF Grant DMS-8702365.

The second author's research was supported in part by The Sloan Foundation and in part by NSF Grant DMS-8722998. 
"exotic" underlying contact structures are generally nonembeddable. See [E] and the references therein.

In this note, we consider perturbations of the standard structure on the three sphere. We show that, in a rather precise sense, the "generic" perturbation is nonembeddable. Our result (4.54) allows for considerable latitude in the choice of topology. In addition to the standard $\mathscr{C}^{\infty}$-topology, our result applies to various topologies on spaces of real analytic perturbations. In (5.3) we give a sufficient condition for embeddability. In (5.27) we show that structures which are infinitesimally obstructed cannot be embedded as 'small perturbations' of the standard sphere.

The standard CR-structure on the three sphere is given by the complex vector field:

$$
\overline{\mathscr{Z}}=w \partial_{\bar{z}}-z \partial_{\bar{w}}
$$

We will let $2 i \mathscr{T}=[\overline{\mathscr{Z}}, \mathscr{Z}]$.

There are no integrability conditions in three dimensions. Thus any complex vector field, $\mathscr{W}$ on $\mathbb{S}^{3}$, such that

$$
[\overline{\mathscr{W}}, \mathscr{W}] \text { is nonvanishing } \bmod \operatorname{sp}\{\mathscr{W}, \overline{\mathscr{W}}\},
$$

defines a strictly pseudoconvex $\mathrm{CR}$-structure on $\mathbb{S}^{3}$. A given structure is homotopic to $\mathscr{Z}$ if it can be joined to the standard structure by a smooth, one parameter family $\mathscr{W}_{l}$, of nondegenerate structures. One can then normalize so that:

$$
\overline{\mathscr{W}}^{\phi} \underset{\operatorname{def}}{=} \overline{\mathscr{Z}}+\phi \mathscr{Z}
$$

Here $\phi \in \mathscr{C}^{\infty}\left(\mathbb{S}^{3}\right)$ with sup-norm less than one. With this normalization

$$
\left[\overline{\mathscr{W}}^{\phi}, \mathscr{W}^{\phi}\right]=2 i\left(1-|\phi|^{2}\right) \mathscr{T} \bmod T^{1,0}+T^{0,1} \text {. }
$$

The ambiguity in this parametrization arises from the action of the group of contact diffeomorphisms of $\mathbb{S}^{3}$. If $\Psi$ is such a map then

$$
\Psi_{*}\left(\overline{\mathscr{W}}^{\phi_{1}}\right)=e^{f} \overline{\mathscr{W}}^{\phi_{2}} \text { for some } f \in \mathscr{C}^{\infty}\left(\mathbb{S}^{3}\right) \text {. }
$$

$\overline{\mathscr{W}}^{\phi_{1}}$ and $\overline{\mathscr{W}}^{\phi_{2}}$ define equivalent CR-structures. For the most part we will not address the question of constructing a local moduli space. Recently, Jack Lee and J. H. Cheng constructed a local slice for this group action, [L]. Every structure near to a given structure appears in this slice and is represented by, at most, a finite-dimensional subvariety, see $\S 4$.

Let $\omega$ denote the $(1,0)$-form dual to $\mathscr{Z}$, relative to the frame $\{\mathscr{Z}, \bar{Z}, \mathscr{T}\}$. The associated $\bar{\partial}_{b}$-operator is given by

$$
\bar{\partial}_{b}^{\phi} f=\overline{\mathscr{W}}^{\phi} f(\bar{\omega}-\bar{\phi} \omega) /\left(1-|\phi|^{2}\right) .
$$

Using the standard metric on $\mathbb{S}^{3}$ to define adjoints, we define

$$
\square_{b}=\bar{\partial}_{b}^{*} \bar{\partial}_{b} \text {. }
$$


A simple calculation shows that the $\square_{b}$-operator defined by $\overline{\mathscr{W}}^{\phi}$ is given by:

$$
\square_{b}^{\phi} f=-(\mathscr{Z}+\overline{\mathscr{Z}} \bar{\phi}) \frac{1+|\phi|^{2}}{\left(1-|\phi|^{2}\right)^{2}}(\overline{\mathscr{Z}}+\phi \mathscr{Z}) f .
$$

Our proof of generic nonembeddability relies on the following theorem of Kohn and Boutet de Monvel. It connects the question of embeddability with the analytic properties of the $\bar{\partial}_{b}$-operator:

Theorem 0.6 (see [Ko, B]). An abstract, strictly pseudoconvex CR-structure on a compact manifold is embeddable if and only if the $L^{2}$-closure of the associated $\bar{\partial}_{b}$-operator has a closed range.

As a simple application of the closed graph theorem, one can show that $\bar{\partial}_{b}$ has a closed range if and only if $\square_{b}$ has a closed range. In $\S \S 3-4$ we consider one parameter families of CR-structures of the form $\overline{\mathscr{W}}^{t \phi}$. The associated $\square_{b}$-operators form an analytic family. As a consequence of this fact, we obtain a sufficient condition which implies that, for a dense open subset of parameter values, the structures, $\overline{\mathscr{W}}^{t \phi}$, are nonembeddable. This condition is satisfied by "generic" functions $\phi \in \mathscr{C}^{\infty}\left(\mathbb{S}^{3}\right)$ or $\mathscr{C}^{\omega}\left(\mathbb{S}^{3}\right)$.

Our sufficient condition for embeddability is simply a support condition for the Fourier coefficients of the function $\phi$. We embed the structure $\overline{\mathscr{W}}^{\phi}$ as a perturbation of the standard structure by finding functions, $\eta$ and $\xi$, so that:

$$
\overline{\mathscr{W}}^{\phi}(w+\eta)=\overline{\mathscr{W}}^{\phi}(z+\xi)=0 .
$$

For which

$$
(w, z) \longrightarrow(w+\eta, z+\xi)
$$

defines an embedding of $\mathbb{S}^{3}$. To accomplish this we rephrase $(0.7)$ as a fixed point problem; the conditions on $\phi$ assure that the successive iterates lie in the range of $\bar{Z}$. Conversely, we show that if certain Fourier coefficients of $\phi$ are nonzero, then at least one of the equations in (0.7) does not have a 'small' solution.

In $\S 1$ we consider the spectral theory of $\square_{b}$-operators. The main result is that $\operatorname{spec}\left(\square_{b}\right) \backslash\{0\}$ consists of point eigenvalues of finite multiplicity. In $\S 2$ we show that the operator $\square_{b}^{\phi}$ is a real analytic function of $\Re \phi$ and $\Im \phi$. This is somewhat involved as the domain of $\square_{b}^{\phi}$ depends on $\phi$. Therefore, standard analytic perturbation theory does not apply. Instead we use the "Heisenberg Calculus" described in [BG] to construct a parametrix for the resolvent. It is manifestly real analytic in the functional parameters.

In $\S 3$ we compute the first two derivatives of the analytic family of compact operators

$$
-\mathscr{A}_{t}=\square_{b}^{t \phi} \mathbf{E}_{\left[0, \frac{1}{2}\right]^{\prime \phi}} .
$$

Here $\mathbf{E}_{[0, \lambda]}^{\psi}$ is the family of spectral projections defined by $\square_{b}^{\psi}$. In $\S 4$ we prove the generic nonembeddability result. 
As a consequence of our analysis one sees that the mapping:

$$
\mathscr{A}(\phi): \phi \longrightarrow \square_{b}^{\phi} \mathbf{E}_{[0, \lambda]}^{\phi}
$$

is, in some sense, an "analytic" mapping. The space of embeddable structures is a stratified space with strata:

$$
\mathfrak{S}_{n}=\{\phi: \mathrm{rk} \mathscr{A}(\phi) \leq n\} .
$$

It is a simple consequence of the proof of (2.18) that $\phi \in \mathfrak{S}_{0}$, sufficiently close to 0 , represents a small perturbation of the unit sphere in $\mathbb{C}^{2}$. An important outstanding question, at least for perturbations of the sphere, is whether the strata stabilize after a finite $n$. In fact, $\mathfrak{S}_{n}$ may equal $\mathfrak{S}_{0}$ for $n>0$.

\section{THE SPECTRAL THEORY OF $\square_{b}$}

The results in this section apply to any strictly pseudoconvex, CR-structure on a 3 -manifold, $M$. If we choose a vector field, $\mathscr{T}$ transverse to $T^{0,1}+T^{1,0}$, then we can define $\bar{\partial}_{b}$ as a differential operator $\bar{\partial}_{b} f=\overline{\mathscr{W}} f \bar{\omega}$. Here $\overline{\mathscr{W}}$ is a local section of $T^{0,1}, \bar{\omega}$ is normalized by $\bar{\omega}(\overline{\mathscr{W}})=1, \bar{\omega}(\mathscr{W})=0, \bar{\omega}(\mathscr{T})=0$. We assume that there is a Riemannian metric $\langle\cdot, \cdot\rangle$ defined on $M$. This, in turn, defines an hermitian $L^{2}$-structure on sections of $T^{*}(M) \otimes \mathbb{C}$ and the adjoint of $\bar{\partial}_{b}$. Formally $\square_{b}=\bar{\partial}_{b}^{*} \bar{\partial}_{b}$. To define the $L^{2}$-closure we employ the Friedrichs' extension method, using the quadratic form

$$
Q(f)=\int_{M}\left\langle\bar{\partial}_{b} f, \bar{\partial}_{b} f\right\rangle \mathrm{dVol} \text {. }
$$

Denote the $L^{2}$-closure by $\mathbf{a}_{b}$. It is a standard result, see [Ka], that

$$
\operatorname{Dom}\left(\boldsymbol{\square}_{b}\right)=\left\{f \in L^{2}(M) ; \bar{\partial}_{b} f \in L^{2}\left(M ; \Lambda^{0,1}\right) \text { and } \bar{\partial}_{b}^{*} \bar{\partial}_{b} f \in L^{2}(M)\right\} \text {. }
$$

The derivatives in (1.2) are taken in the sense of distributions.

The quadratic form $Q$ is nonnegative and hermitian symmetric so the operator $\boldsymbol{\square}_{b}$ is nonnegative and selfadjoint. Thus, the $\operatorname{spec}\left(\boldsymbol{\square}_{b}\right)$ lies in $[0, \infty)$. The following theorem shows that, away from zero, the spectrum of the operator is quite simple.

Theorem 1.3. The $\operatorname{spec}\left(\boldsymbol{G}_{b}\right)$ in $(0, \infty)$ consists of point eigenvalues of finite multiplicity.

Proof. To prove the theorem, we use the results in [BG] on the construction of an approximate "Szegö"-projector and a partial inverse to $\square_{b}$. In particular, they construct an operator $\mathscr{S} \in S_{\frac{1}{2}, \frac{1}{2}}^{0}(M)$ such that:

$$
\square_{b} \mathscr{S}=K_{1} \in S_{\frac{1}{2}, \frac{1}{2}}^{-\infty}(M)
$$

and an operator $\mathscr{Q}$ in $S_{\frac{1}{2}, \frac{1}{2}}^{-1}(M)$ such that:

$$
\square_{b} \mathscr{Q}-\mathrm{Id}+\mathscr{S}=K_{2} \in S_{\frac{1}{2}, \frac{1}{2}}^{-\frac{1}{2}}(M) \text {. }
$$


The theorem of Calderon-Villaincourt implies that the operators $\mathscr{Q}, K_{1}$, and $K_{2}$ are compact on $L^{2}$. We obtain a parametrix for the resolvent by setting

$$
R^{\prime}(\lambda)=-\mathscr{S} / \lambda+\mathscr{Q} \text {. }
$$

From (1.4) and (1.5) we obtain

$$
\left(\square_{b}-\lambda\right) R^{\prime}(\lambda)=\left(\operatorname{Id}+K_{2}-K_{1} / \lambda-\lambda \mathscr{Q}\right)=E(\lambda) \text {. }
$$

From the compactness of $\mathscr{Q}, K_{1}$, and $K_{2}$ it follows that $E(\lambda)$ is an analytic, Fredholm family in $\mathbb{C} \backslash\{0\}$. We may assume it is invertible for some $\lambda$.

Suppose this were not the case. Since $\square_{b}$ is a nonnegative operator it follows that $\square_{b}+1$ is invertible. We rewrite $(1.7)$ as

$$
\left(\square_{b}+1\right) R^{\prime}(1)=\mathrm{Id}+K \text {. }
$$

As $K$ is an operator of order $-\frac{1}{2}$, the right-hand side of (1.8) is a Fredholm operator of index zero. Let $\left\{u_{1}, \ldots, u_{m}\right\}$ be an orthonormal basis for $\operatorname{null}(\operatorname{Id}+K)$ and $\left\{v_{1}, \ldots, v_{m}\right\}$ be an orthonormal basis for null(Id $\left.+K^{*}\right)$. Since $K$ and $K^{*}$ are operators of order $-\frac{1}{2}$, it is clear that the null spaces of Id $+K$ and $\operatorname{Id}+K^{*}$ are contained in $\mathscr{C}^{\infty}(M)$. Since $\square_{b}+1$ is invertible, we can find $\left\{w_{1}, \ldots, w_{m}\right\}$ such that

$$
\left(\square_{b}+1\right) w_{i}=v_{i}
$$

The operator $\mathscr{S}^{*}+\mathscr{Q}$ is a left parametrix for $\square_{b}+1$ with an error in $S_{\frac{1}{2}, \frac{1}{2}}^{-\frac{1}{2}}(M)$. Hence it follows that $w_{i} \in \mathscr{C}^{\infty}(M), i=1, \ldots, m$, as well. If we modify $\mathscr{Q}$ by adding the finite rank, $\mathscr{C}^{\infty}$ kernel

$$
C=\sum_{i=1}^{m} w_{i} \otimes u_{i}
$$

then we obtain

$$
\left(\square_{b}+1\right)(\mathscr{S}+\mathscr{Q}+C)=\left(\mathrm{Id}+K+\sum_{i=1}^{m} v_{i} \otimes u_{i}\right) .
$$

The right-hand side of $(1.10)$ is clearly invertible. By replacing $\mathscr{Q}$ with $\mathscr{Q}+C$, we may assume that $E(\lambda)$ of (1.7) is invertible on some open set, hence, in all but a discrete subset of $\mathbb{C} \backslash\{0\}$. The theorem follows immediately from this.

The proof of the theorem has the following immediate corollary:

Corollary 1.11. The eigenfunctions of $\square_{b}$ corresponding to nonzero eigenvalues are in $\mathscr{C}^{\infty}(M)$.

It is a well-known "folk theorem" that no local calculation can distinguish an embeddable CR-structure from a nonembeddable one. The range of $\boldsymbol{a}_{b}$ is closed if and only if 0 is an isolated eigenvalue. As a consequence, one would expect that the "small eigenvalues" of $\mathbf{b}_{b}$ form a rapidly decreasing sequence. This is in fact the case. 
Proposition 1.12. Let $\left\{1 \geq \mu_{1} \geq \mu_{2} \geq \cdots\right\}$ denote the sequence of small eigenvalues of $\boldsymbol{a}_{b}$. Then for any $N>0$, there exists a constant, $C_{N}$, such that

$$
\mu_{m} \leq C_{N} / m^{N} .
$$

Proof. Suppose that $r$ is a small positive number, not in the spectrum of $\boldsymbol{\Xi}_{b}$. Let $R(\lambda)$ denote the resolvent of $\boldsymbol{a}_{b}$. Define

$$
-2 \pi i A=\int_{|\lambda|=r} \mathbf{\Xi}_{b} R(\lambda) d \lambda=\int_{|\lambda|=r} \lambda R(\lambda) d \lambda .
$$

Since $\operatorname{spec}(A)=\left\{\mu_{n}, \mu_{n+1}, \ldots\right\}$, for some finite $n$, it suffices to prove that $A$ is an integral operator with a smooth kernel. Recall that the resolvent can be written as

$$
R(\lambda)=R^{\prime}(\lambda) E(\lambda)^{-1} .
$$

We can rewrite $E(\lambda)$ of (1.7) in a manner which shows that the spectrum of $\boldsymbol{\Xi}_{b}$, near to zero, is a rapidly decreasing sequence. The operator $I+K_{2}$ is Fredholm of index zero. We can find a finite rank operator $C^{\prime}$, with smooth kernel such that $\mathrm{Id}+K_{2}+C^{\prime}$ is invertible. We then obtain

$$
\begin{aligned}
& E(\lambda)=\left(\mathrm{Id}-K_{\lambda} / \lambda\right) L_{\lambda} \\
& \quad K_{\lambda}=\left(K_{1}+\lambda C^{\prime}\right)\left(\mathrm{Id}+K_{2}+C^{\prime}-\lambda \mathscr{Q}\right)^{-1} \text { and } L_{\lambda}=\left(\mathrm{Id}+K_{2}+C^{\prime}-\lambda \mathscr{Q}\right) .
\end{aligned}
$$

Since $K_{1}$ and $C^{\prime}$ belong to $S^{-\infty}(M)$, the kernel of $K_{\lambda}$ is an analytic function of $\lambda$ with values in $\mathscr{C}^{\infty}(M \times M)$.

For sufficiently small $r, L_{\lambda}^{-1}$ is bounded and analytic in $D_{r}(0)$. Moreover, we can choose a small $r$ such that $\left(\operatorname{Id}-K_{\lambda} / \lambda\right)^{-1}$ exists for $|\lambda|=r$. Denote this radius by $r_{0}$. Using the Neumann series, it is simple to show that:

$$
\left(\operatorname{Id}-K_{\lambda} / \lambda\right)^{-1}=\operatorname{Id}-K_{\lambda}^{\prime} ; \quad \text { where } K_{\lambda}^{\prime} \in \mathscr{C}^{\infty}(M \times M),|\lambda|=r_{0} .
$$

It follows from (1.15)-(1.17) that we can write

$$
\lambda R(\lambda)=M(\lambda)-M(\lambda) K_{\lambda}^{\prime} .
$$

Here $M(\lambda) \in S_{\frac{1}{2}, \frac{1}{2}}^{0}(M)$ is analytic in $D_{r_{0}}(0)$. Thus the integral in (1.14) reduces to

$$
2 \pi i A=\int_{|\lambda|=r_{0}} M(\lambda) K_{\lambda}^{\prime} d \lambda .
$$

Along the path of integration, the integrand in (1.19) is analytic with values in $\mathscr{C}^{\infty}(M \times M)$. From this it follows that $A$ has a kernel in $\mathscr{C}^{\infty}(M \times M)$ as well. The proposition follows immediately from this. 


\section{THE ANALYTICITY PROPERTIES OF FAMILIES}

The main result of this section is

Theorem 2.1. Let $\phi_{1}(t)$ and $\phi_{2}(t)$ be real analytic functions from $D_{\varepsilon}(0) \subset \mathbb{C}$ into $C^{k}\left(\mathbb{S}^{3}\right)$, for a sufficiently large $k$. Suppose that $\phi_{i}(0)=0, i=1,2$ and set

$$
\phi(t)=\phi_{1}(t)+i \phi_{2}(t) .
$$

The family of operators $t \rightarrow \mathbf{\square}_{b}^{\phi(t)}$ is analytic, in the strong resolvent sense, for $t$ in a sufficiently small neighborhood of 0 .

Remark. As the constructions using the Heisenberg calculus are local, an analogous theorem holds for perturbations of a CR-structure on any three-dimensional manifold.

Proof. The proof follows from a careful study of the dependence on parameters in the method of [BG] for constructing approximate partial inverses and projectors for $\square_{b}$ operators. For the benefit of the reader, we will outline their construction in this special case. The basis of their analysis is the introduction of a calculus for an algebra of pseudodifferential operators, $S_{\mathscr{V}}^{*}\left(\mathbb{S}^{3}\right)$. Here and in the sequel $\mathscr{V}=T^{1,0}\left(\mathbb{S}^{3}\right)+T^{0,1}\left(\mathbb{S}^{3}\right)$. The symbol classes satisfy:

$$
S_{\mathscr{V}}^{m} \subset S_{\frac{1}{2}, \frac{1}{2}}^{m} \text { if } m \geq 0 \text { and } S_{\mathscr{V}}^{m} \subset S_{\frac{1}{2}, \frac{1}{2}}^{\frac{1}{2} m} \text { if } m \leq 0 .
$$

Unlike the $\left(\frac{1}{2}, \frac{1}{2}\right)$-symbol classes, the operators in $S_{\mathscr{V}}^{*}$ have a full symbol calculus with a good composition formula. The boundedness properties, on $L^{2}$ Sobolev spaces, follow from (2.2).

The method of Beals and Greiner is, roughly speaking, to find "model problems" that osculate the given operator to the highest possible order at each point. One then constructs approximate kernels by using the kernels for the model problems, coordinate transformations, and the smooth dependence on parameters.

In the case of a strongly pseudoconvex, three-dimensional CR-manifold there is a unique model problem provided by the hyperquadric in $\mathbb{C}^{2}$ :

$$
\mathbb{Q}=\left\{(w, z) ; \Im w=\frac{1}{2} z \bar{z}\right\} .
$$

The underlying group structure is the Heisenberg group. Let $u, x, y$ denote coordinates for $\mathbb{R}^{3}$ then

$$
\mathbb{Q}=\left\{u+\frac{i}{2}\left(x^{2}+y^{2}\right), x+i y\right\} .
$$

The group composition law is

$$
\left(u_{1}, x_{1}, y_{1}\right) \cdot\left(u_{2}, x_{2}, y_{2}\right)=\left(u_{1}+u_{2}+x_{2} y_{1}-x_{1} y_{2}, x_{1}+x_{2}, y_{1}+y_{2}\right) \text {. }
$$

The vector fields:

$$
X=\frac{1}{2}\left(\partial_{x}+y \partial_{u}\right), \quad Y=\frac{1}{2}\left(\partial_{y}-x \partial_{u}\right), \quad T=\frac{1}{2} \partial_{u}
$$


are left invariant with respect to the above group structure and satisfy:

$$
[X, Y]=-T \text {. }
$$

The vector fields $X$ and $Y$ belong to $S_{\mathscr{V}}^{1}, T$ belongs to $S_{\mathscr{V}}^{2}$.

In terms of these coordinates, the standard CR-structure for the hyperquadric is given by $\overline{\mathscr{W}}=X+i Y$. The standard $\square_{b}$-operator is simply $\square_{b}^{0}=-\mathscr{W} \overline{\mathscr{W}}$. In addition to the group structure, there is also a dilation structure on $\mathbb{R}^{3}$, crucial in the analysis of such operators:

$$
M_{\delta}(u, x, y)=\left(\delta^{2} u, \delta x, \delta y\right) .
$$

The projector onto the null space of $\square_{b}^{0}$ is a convolution operator with respect to the above group structure. The kernel is given by:

$$
S_{0}(u, x, y)=\frac{1}{2 \pi^{2}\left(\frac{1}{2}\left(x^{2}+y^{2}\right)-i u\right)^{2}} .
$$

The kernel is homogeneous of degree -4 with respect to $M_{\delta}$. Thus it defines an element of $S_{\mathscr{V}}^{0}(\mathbb{Q})$. The partial inverse for $\square_{b}^{0}$ is also a convolution operator and its kernel is given by

$$
Q_{0}(u, x, y)=\frac{-2}{\pi^{2}} \int_{-\infty}^{\infty} \frac{s\left(\frac{1}{2} \cosh (2 s)\left(x^{2}+y^{2}\right)+i e^{-2 s} u\right) d s}{\cosh ^{3}(2 s)\left(\frac{1}{2}\left(x^{2}+y^{2}\right)-i \tanh (2 s) u\right)^{2}}
$$

This kernel is homogeneous of order -2 and therefore defines an element of $S_{\mathscr{V}}^{-2}(\mathbb{Q})$.

To construct approximate projectors and partial inverses for a general $\square_{b}$ operator, one composes these kernels with the coordinate changes that put the operator into normal form at each point. For the purposes of local computations, it is much simpler to work in the $\mathbb{R}^{3}$ model than on the three sphere or the hyperquadric.

The sphere is covered by two "holomorphic" coordinate patches. The maps from the sphere to the hyperquadric are given by:

$$
w=i \frac{1 \pm \eta}{1 \mp \eta}, \quad z=\frac{2^{\frac{1}{2}} \xi}{1 \mp \eta} .
$$

The vector field $\bar{Z}$, given in $(0.1)$, is transformed to a vector field of the form $\bar{m} \overline{\mathscr{W}}$. The operator $\square_{b}^{\phi}$, defined in $(0.5)$, is given in the coordinates $u, x, y$ by

$$
\square_{b}^{\phi}=-(m \mathscr{W}+\bar{m} \overline{\mathscr{W}} \bar{\phi}) \frac{\left(1+|\phi|^{2}\right)}{\left(1-|\phi|^{2}\right)^{2}}(\bar{m} \overline{\mathscr{W}}+\phi m \mathscr{W}) .
$$

This operator belongs to $S_{\mathscr{V}}^{2}\left(\mathbb{R}^{3}\right)$, we can rewrite it as

$$
\square_{b}^{\phi}=-M\left(\mathscr{W}+\frac{\bar{m}}{\bar{m}} \phi \overline{\mathscr{W}}\right) M\left(\overline{\mathscr{W}}+\frac{m}{\bar{m}} \phi \mathscr{W}\right)+P_{1}
$$


Here

$$
M=\frac{|m|\left(1+|\phi|^{2}\right)^{\frac{1}{2}}}{1-|\phi|^{2}}, \quad P_{1} \in S_{\mathscr{V}}^{1}\left(\mathbb{R}^{3}\right) .
$$

It is important to note that the coefficients of $P_{1}$ are rational functions of $\phi_{1}, \phi_{2}$, and a finite number of their derivatives. We now construct kernels for operators that are the leading order terms for the approximate projector and partial inverse for $\square_{b}^{\phi}$. The real analytic dependence on $\phi_{1}$ and $\phi_{2}$ is manifest from the construction. To simplify notation we set $\psi_{1}+i \psi_{2}=\frac{m}{m} \phi$. In order to find the model operators, we need to write the principal part as a sum of squares:

$$
\begin{gathered}
\square_{b \mathrm{pr}}^{\phi}=A^{2}+B^{2}-i C+P_{1}^{\prime} \quad \text { where } A=M\left(\left(1+\psi_{1}\right) X+\psi_{2} Y\right), \\
B=M\left(\left(1-\psi_{1}\right) Y+\psi_{2} X\right), C=M^{2}\left(1-\psi_{1}^{2}-\psi_{2}^{2}\right)[X, Y] .
\end{gathered}
$$

$P_{1}^{\prime}$ is another error term, lying in $S_{\mathscr{V}}^{1}\left(\mathbb{R}^{3}\right)$, with rational dependence on $\psi_{1}$ and $\psi_{2}$.

The next step in constructing the normal form at a point $p$ is to apply the left translation by $p^{-1}$. The point of interest becomes the origin. Denote the translated coordinates by $(\tilde{u}, \tilde{x}, \tilde{y})$. The principal part retains the same form as the vector fields $X$ and $Y$ are left invariant. Ne't we apply a linear transformation so that in the new coordinates, $(a, b, c)$, the vector fields $A, B, C$ agree to first order at $(0,0,0)$ with

$$
A^{\prime}=\frac{1}{2}\left(\partial_{b}+c \partial_{a}\right), \quad B^{\prime}=\frac{1}{2}\left(\partial_{c}-b \partial_{a}\right), \quad C^{\prime}=\frac{1}{2} \partial_{a} .
$$

The transformation is given by:

$$
\begin{aligned}
& a=-\frac{\tilde{u}}{M^{2}\left(1-\psi_{1}^{2}-\psi_{2}^{2}\right)}, \\
& b=\frac{\left(1-\psi_{1}\right) \tilde{x}-\psi_{2} \tilde{y}}{M\left(1-\psi_{1}^{2}-\psi_{2}^{2}\right)}, \\
& c=-\frac{\psi_{2} \tilde{x}-\left(1+\psi_{1}\right) \tilde{y}}{M\left(1-\psi_{1}^{2}-\psi_{2}^{2}\right)}
\end{aligned}
$$

In (2.14) $\psi_{1}, \psi_{2}$, and $M$ are evaluated at $p$. We will denote this transformation by $T_{p}(\tilde{u}, \tilde{x}, \tilde{y})$. If $\psi_{1}$ and $\psi_{2}$ are real then this is precisely the construction carried out in [BG]. The leading order terms in the approximate projector and partial inverse are given by

$$
\mathscr{Q}_{0}^{\phi}(p, q)=\frac{Q_{0}\left(T_{p}\left(p^{-1} \cdot q\right)\right) d u d x d y}{M^{4}\left(1-\psi_{1}^{2}-\psi_{2}^{2}\right)^{2}}
$$

and

$$
\mathscr{S}_{0}^{\phi}(p, q)=\frac{S_{0}\left(T_{p}\left(p^{-1} \cdot q\right)\right) d u d x d y}{M^{4}\left(1-\psi_{1}^{2}-\psi_{2}^{2}\right)^{2}}
$$


The kernels $\mathscr{Q}_{0}^{\phi}$ and $\mathscr{S}_{0}^{\phi}$ define elements of $S_{\mathscr{V}}^{-2}(V)$ and $S_{\mathscr{V}}^{0}(V)$ respectively. Here $V$ is a neighborhood of 0 in $\mathbb{R}^{3}$. This follows from Theorem 15.49 in [BG] as $Q_{0}$ and $S_{0}$ are homogeneous with respect to $M_{\delta}$ and have their singular supports contained in $\{(0,0,0)\}$. Moreover $S_{0}$ has average 0 over Carnot spheres about $(0,0,0)$. The main step towards proving the analyticity in $\phi_{1}$ and $\phi_{2}$ is to see that the singular supports of $Q_{0}\left(T_{p}(q)\right)$ and $S_{0}\left(T_{p}(q)\right)$, for $q \in \mathbb{R}^{3}$, remain fixed at $(0,0,0)$ when the $\Im \psi_{1}$ and $\Im \psi_{2}$ are small but nonzero. From (2.7), (2.8) it is clear that the singular supports lie in the intersection of $T_{p}\left(\mathbb{R}^{3}\right)$ with the varieties $N_{s}=\left\{(a, b, c) ; b^{2}+c^{2}=i s a, s \in \mathbb{R} \backslash\{0\}\right\}$. These intersections are the real solutions of the equation:

$$
\frac{\left(\left(1-\psi_{1}\right) \tilde{x}-\psi_{2} \tilde{y}\right)^{2}+\left(\psi_{2} \tilde{x}-\left(1+\psi_{1}\right) \tilde{y}\right)^{2}}{1-\psi_{1}^{2}-\psi_{2}^{2}}=i s \tilde{u} .
$$

An elementary calculation shows that only $(\tilde{u}, \tilde{x}, \tilde{y})=(0,0,0)$ solves $(2.17)$ if $\Im \psi_{1}$ and $\Im \psi_{2}$ are sufficiently small.

Altogether we have shown

Proposition 2.18. If $\Im \psi_{1}$ and $\Im \psi_{2}$ are sufficiently small then the kernels $\mathscr{Q}_{0}^{\phi}$ and $\mathscr{S}_{0}^{\phi}$ given by (2.15) and (2.16) define operators in $S_{\mathscr{V}}^{-2}(V)$ and $S_{\mathscr{V}}^{0}(V)$ respectively. $V$ is some neighborhood of $(0,0,0) \in \mathbb{R}^{3}$. In this neighborhood we also have:

$$
\begin{aligned}
\square_{b}^{\phi} \mathscr{Q}_{0}^{\phi}-\mathrm{Id}+\mathscr{S}_{0}^{\phi} & \in S_{\mathscr{V}}^{-1}(V), \\
\square_{b}^{\phi} \mathscr{S}_{0}^{\phi} & \in S_{\mathscr{V}}^{1}(V) .
\end{aligned}
$$

The error terms in (2.19) are rational functions of $\phi_{1}, \phi_{2}$, and finitely many of their derivatives.

In order to obtain a parametrix for $\square_{b}^{\phi}$ we need to modify $\mathscr{S}_{0}^{\phi}$ to obtain a compact error. In $\S 25$ of [BG] an algorithm is presented to accomplish precisely this task. They construct the adjoint of $\mathscr{S}^{\phi}$, as a kernel of the form:

$$
\mathscr{S}^{\phi *}(p, q)=S^{*}\left(p, T_{p}\left(p^{-1} \cdot q\right)\right) \text {. }
$$

$S^{*}$ is obtained as a sum of homogeneous terms:

$$
S^{*} \sim S_{0}^{*}+\sum_{k=1}^{\infty} S_{-k}^{*}, \quad S_{-k}^{*} \in S_{\mathscr{V}}^{-k}(V) .
$$

If $\vartheta_{b}=\bar{\partial}_{b}^{\phi *}$ is expanded as a sum of homogeneous terms

$$
\vartheta_{b} \sim \vartheta_{b ; 1}+\sum_{k=0}^{\infty} \vartheta_{b ;-k},
$$

then each term on the right-hand side of (2.21) is a rational function of 
$\phi_{1}, \phi_{2}$, and finitely many of their derivatives. The terms in (2.20) are given by:

$$
\begin{aligned}
S_{-k-1}^{*} & =\sum_{j=0}^{k} S_{-j}^{*} \vartheta_{b ; j-k} W, \\
\text { where } W & =\frac{x+i y}{2 \pi^{2}\left(\frac{1}{2}\left(x^{2}+y^{2}\right)-i u\right)^{2}\left(\frac{1}{2}\left(x^{2}+y^{2}\right)+i u\right)^{2}} .
\end{aligned}
$$

Thus $S^{*}$ depends rationally on $\phi$ and is a sum of homogeneous terms. All we need to check is the singular support of the kernels in (2.22) when $\Im \psi_{1}$ and $\Im \psi_{2}$ are small but nonzero. It follows easily from (2.17) and the form of $W$ that this remains fixed at $(0,0,0)$. If we let

$$
\widetilde{\mathscr{S}}^{\phi *}(p, q)=\sum_{j=0}^{2} S_{-j}^{*}\left(p, T_{p}\left(p^{-1} \cdot q\right)\right),
$$

then

$$
\square_{b}^{\phi} \widetilde{\mathscr{S}}^{\phi} \in S_{\mathscr{V}}^{-1}(V) \text { and } \mathscr{S}_{0}^{\phi}-\widetilde{\mathscr{S}}^{\phi} \in S_{\mathscr{V}}^{-1}(V) .
$$

Moreover, $\widetilde{\mathscr{S}}^{\phi}$ depends rationally on $\phi_{1}, \phi_{2}$, and finitely many of their derivatives.

If we set

$$
\widetilde{R}^{\phi}(\lambda)=-\widetilde{\mathscr{S}}^{\phi} / \lambda+\mathscr{Q}_{0}^{\phi}
$$

then

$$
\left(\square_{b}^{\phi}-\lambda\right) \widetilde{R}^{\phi}(\lambda)=\operatorname{Id}-K^{\phi}(\lambda) \quad \text { where } K^{\phi}(\lambda) \in S_{\mathscr{V}}^{-1}(V) .
$$

Moreover $\widetilde{R}^{\phi}$ and $K^{\phi}$ depend rationally on $\phi_{1}, \phi_{2}$, and finitely many of their derivatives. Using a partition of unity, we can obtain the analogous operators on $\mathbb{S}^{3}$. We denote their kernels with the same notation.

The error term in (2.26) can be written more precisely as

$$
\begin{aligned}
& -K^{\phi}(\lambda)=K_{2}^{\phi}-\lambda \mathscr{Q}_{0}^{\phi}-\frac{K_{1}^{\phi}}{\lambda} \\
& \text { where } K_{1}^{\phi}=\square_{b}^{\phi} \widetilde{\mathscr{S}}^{\phi} \text { and } K_{2}^{\phi}=\square_{b}^{\phi} \mathscr{Q}_{0}^{\phi}-\mathrm{Id}+\widetilde{\mathscr{S}}^{\phi} .
\end{aligned}
$$

If $\phi$ tends to zero in $C^{k}\left(\mathbb{S}^{3}\right)$, for sufficiently large $k$, then $K_{1}^{\phi}$ and $K_{2}^{\phi}$ tend uniformly to zero as $\mathscr{Q}_{0}^{\phi}$ and $\widetilde{\mathscr{S}}^{\phi}$ tend to the partial inverse and Szegö projector for the standard structure on $\mathbb{S}^{3}$. Thus, for $\phi$ sufficiently small, in the $C^{k}\left(\mathbb{S}^{3}\right)$ sense, the error term (Id $\left.-K^{\phi}(i)\right)$ is invertible.

From this, (2.26) and the rational dependence on $\phi$, it follows that the inverse of $\boldsymbol{a}_{b}^{\phi}-i$ is a real analytic function of $\phi_{1}, \phi_{2}$, and finitely many of their derivatives. This completes the proof. 
To apply the above result in the construction we need the following general lemmas:

Lemma 2.28. Suppose that $A(z)$ is an analytic family of operators in a domain $D \subset \mathbb{C}$ and that $\mathrm{rk} A(z)$ is finite throughout $D$, then

$$
r_{\max }=\sup _{z \in D} \operatorname{rk} A(z)
$$

is finite. The set $\left\{z ; \operatorname{rk} A(z)<r_{\max }\right\}$ has no accumulation point in $D$.

Proof. Since $A(z)$ depends continuously on $z$ it is a simple matter to prove that the sets $R_{n}=\{z ; \operatorname{rk} A(z) \leq n\}$ are all closed. From the hypothesis of the theorem, it follows that

$$
D=\bigcup_{n \geq 0} R_{n}
$$

It is a simple consequence of the Baire category theorem that at least one of the sets, $R_{n}$, has nonempty interior. There is a smallest value of $n$ for which this is true. Denote it by $n_{0}$. We can suppose that $n_{0}>0$. If $A(z)=0$ on an open set then $A(z)$ is identically zero. In virtue of the fact that $n_{0}$ is the minimum value for which $\stackrel{\circ}{R}_{n} \neq \varnothing, \operatorname{rk} A(z)=n_{0}$ on an open subset $U$ of $R_{n_{0}}$. We can find vectors $\left\{v_{1}, \ldots, v_{n_{0}}\right\}$ and linear functionals $\left\{l_{1}, \ldots, l_{n_{0}}\right\}$ such that the analytic function

$$
d(z)=\operatorname{det}\left(l_{i}\left(A(z) v_{j}\right)\right)
$$

is not identically zero. The set $\{z ; d(z)=0\}$ therefore has no point of accumulation in $D$. Suppose that $\mathrm{rk} A\left(z^{\prime}\right)>n_{0}$. Then we can find vectors $\left\{u_{1}, \ldots, u_{n_{0}+1}\right\}$ and linear functionals $\left\{m_{1}, \ldots, m_{n_{0}+1}\right\}$ such that the analytic function

$$
d^{\prime}(z)=\operatorname{det}\left(m_{i}\left(A(z) u_{j}\right)\right)
$$

does not vanish at $z^{\prime}$. This implies that the function $d^{\prime}(z)$ must be nonzero somewhere on the open set $U$. But this contradicts $\operatorname{rk} A(z)=n_{0}$ for $z \in U$.

We have an analogous result for operators of infinite rank

Lemma 2.31. Suppose that $A(z)$ is an analytic family of bounded operators on a domain $D \subset \mathbb{C}$ and that $A(z)$ has infinite rank at some point of $D$, then the set

$$
\{z \in D ; \text { rk } A(z)<\infty\}
$$

is countable.

Proof. Let $z^{\prime}$ be a point where $A(z)$ has infinite rank. There exist vectors $\left\{u_{1}, \ldots\right\}$ and linear functionals $\left\{l_{1}, \ldots\right\}$ such that if we define

$$
M_{n}(z)=l_{i}\left(A(z) u_{j}\right) \quad \text { where } 1 \leq i, j \leq n,
$$

then $\operatorname{det} M_{n}\left(z^{\prime}\right) \neq 0$ for $n \in \mathbb{N}$. Each of the functions $M_{n}(z)$ is analytic. Thus each subset, $Z_{n}=\left\{z \in D ; \operatorname{det} M_{n}(z)=0\right\}$, is countable. The set, $\{z ;$ rk $A(z)<\infty\}$, lies in the union of the $Z_{n}$, thus proving the lemma. 
For our applications we require a small extension of (2.31):

Lemma 2.33. Suppose that $A(z)$ is an analytic family of bounded operators on a domain $D \subset \mathbb{C}$ and that some derivative of $A(z)$ has infinite rank at some point in $D$, then the set

is countable.

$$
\{z \in D ; \operatorname{rk} A(z)<\infty\}
$$

Proof. It is a simple consequence of the Cauchy integral formula, for vectorvalued analytic functions, that the derivatives of $A(z)$ are analytic families of bounded operators in $D$. The lemma follows easily from this fact and the argument used to prove (2.31).

\section{THE FIRST AND SECOND VARIATIONS}

Our strategy for constructing generically nonembeddable examples is to find a criterion which implies that the rank of $\mathscr{A}_{t}=\square_{b}^{t \phi} \mathbf{E}_{[0, \lambda]}^{t \phi}$, for small positive $\lambda$, is infinite for almost every $t$. It follows from (2.1) and (1.19) that $\mathscr{A}_{t}$ is a bounded analytic family in some neighborhood of 0 . In fact, it follows from (1.3) that $\mathscr{A}_{t}$ is a compact holomorphic family. Consequently, the operators

$$
\mathscr{A}^{[k]}=\partial_{t}^{k} \mathscr{A}_{t} \uparrow_{t=0}
$$

are bounded.

As a first step we consider $\mathscr{A}^{[0]}=\square_{b}^{0} \mathbf{E}_{\left[0, \frac{1}{2}\right]}^{0}$. By normalizing the volume of the three sphere we can assume that the spectrum of $\square_{b}^{0}$ is given by:

$$
\operatorname{spec} \square_{b}^{0}=\{0,1,2, \ldots\} \text {. }
$$

Zero is an eigenvalue of infinite multiplicity. Clearly, $\mathscr{A}^{[0]}=0$. As we shall see, the first derivative $\mathscr{A}^{[1]}$ is also zero but the second derivative $\mathscr{A}^{[2]}$ is generically of infinite rank. It then follows from (2.33) that $\mathscr{A}_{t}$ is generically of infinite rank.

We claim that for $t$ sufficiently close to 0 , the $\operatorname{spec}\left(\mathscr{A}_{t}\right) \cap\left\{|\lambda|=\frac{1}{2}\right\}$ is empty. It follows from the proof of (2.1) that $\mathscr{A}_{t}$ is a compact operator, for $t$ in a neighborhood of 0 . Suppose that $\left\{t_{i}\right\}$ converges to zero and that $\left\{\psi_{i}\right\}$ is a sequence of normalized eigenfunctions:

$$
\square_{b}^{t_{i} \phi} \psi_{i}=\lambda_{i} \psi_{i}, \quad\left\|\psi_{i}\right\|=1, \quad\left|\lambda_{i}\right|=\frac{1}{2} .
$$

It follows from (1.7) that $\left(E^{t_{i} \phi}\right)^{*}\left(\lambda_{i}\right) \psi_{i}=0$. The argument used in the proof of (2.1) implies that $\left\{\psi_{i}\right\}$ is a precompact set in $\mathscr{C}^{\infty}\left(\mathbb{S}^{3}\right)$. Let $\psi^{*}$ denote the limit along a subsequence, for which $\left\{\lambda_{i}\right\}$ converges to $\lambda^{*}$. It satisfies:

$$
\square_{b}^{0} \psi^{*}=\lambda^{*} \psi^{*}, \quad\left\|\psi^{*}\right\|=1,\left|\lambda^{*}\right|=\frac{1}{2} .
$$

However this contradicts (3.2), establishing the claim.

We use the formula for $\mathscr{A}_{t}$ given by $(1.14)$

$$
2 \pi i \mathscr{A}_{t}=\int_{|\lambda|=\frac{1}{2}} \lambda R^{t \phi}(\lambda) d \lambda .
$$


From this formula one easily derives that

$$
\begin{aligned}
& 2 \pi i \mathscr{A}^{[1]}=-\int_{|\lambda|=\frac{1}{2}} \lambda R^{0}(\lambda) B^{1} R^{0}(\lambda) d \lambda, \\
& 2 \pi i \mathscr{A}^{[2]}=\int_{|\lambda|=\frac{1}{2}}\left[2 \lambda R^{0}(\lambda) B^{1} R^{0}(\lambda) B^{1} R^{0}(\lambda)-\lambda R^{0}(\lambda) B^{2} R^{0}(\lambda)\right] d \lambda .
\end{aligned}
$$

Here

$$
B^{1}=\partial_{t} \square_{b}^{t \phi} \uparrow t=0 \text { and } B^{2}=\partial_{t}^{2} \square_{b}^{t \phi} \Gamma_{t=0} .
$$

These derivatives are given by

$$
B^{1}=-[\overline{\mathscr{Z}} \bar{\phi} \bar{Z}+\mathscr{Z} \phi \mathscr{Z}], \quad B^{2}=-2\left[\overline{\mathscr{Z}}|\phi|^{2} \mathscr{Z}+3 \mathscr{Z}|\phi|^{2} \overline{\mathscr{Z}}\right] .
$$

The resolvent for $\square_{b}^{0}$ has an expansion at $\lambda=0$ :

$$
R^{0}(\lambda)=-\mathscr{S}_{0} / \lambda+\mathscr{Q}_{0}+O(\lambda)
$$

Here $\mathscr{S}_{0}$ is the orthogonal projection onto the null space of $\square_{b}^{0}$ and $\mathscr{Q}_{0}$ is the partial inverse defined on $\left\{\text { range } \mathscr{S}_{0}\right\}^{\perp}$.

Using these formulae, we easily derive

$$
\mathscr{A}^{[1]}=\mathscr{S}_{0}[\overline{\mathscr{Z}} \bar{\phi} \bar{Z}+\mathscr{Z} \phi \mathscr{Z}] \mathscr{S}_{0} \text {. }
$$

Since $\mathscr{S}_{0}$ is the projector onto the null space of $\overline{\mathscr{Z}}$ it is obvious that $\bar{Z} \mathscr{S}_{0}=0$. As $\mathscr{S}_{0}$ is selfadjoint and $\overline{\mathscr{Z}}^{*}=-\mathscr{Z}$ we also have that $\mathscr{S}_{0} \mathscr{Z}=0$. From these facts and (3.7) we obtain

Lemma 3.8. The first derivative of the family $\mathscr{A}_{t}$ at $0, \mathscr{A}^{[1]}$, vanishes.

From (3.4) and (3.6) we obtain that:

$$
\mathscr{A}^{[2]}=2\left[\mathscr{S}_{0} B^{1} \mathscr{S}_{0} B^{1} \mathscr{Q}_{0}+\mathscr{S}_{0} B^{1} \mathscr{Q}_{0} B^{1} \mathscr{S}_{0}+\mathscr{Q}_{0} B^{1} \mathscr{S}_{0} B^{1} \mathscr{S}_{0}\right]-\mathscr{S}_{0} B^{2} \mathscr{S}_{0} \text {. }
$$

Using the facts that $\mathscr{S}_{0} B^{1} \mathscr{S}_{0}=0, \bar{Z} \mathscr{S}_{0}=0$, and (3.5) we obtain that

$$
\mathscr{A}^{[2]}=2\left[\mathscr{S}_{0} \overline{\mathscr{Z}} \bar{\phi} \overline{\mathscr{Q}} \mathscr{Q}_{0} \mathscr{Z} \phi \mathscr{Z} \mathscr{S}_{0}+\mathscr{S}_{0} \overline{\mathscr{Z}}|\phi|^{2} \mathscr{Z}_{0}\right] \text {. }
$$

Employing the spanning set,

$$
\left\{z^{p} w^{q} \bar{z}^{r} \bar{w}^{s} ; p, q, r, s \in \mathbb{N}_{0}\right\}
$$

for $L^{2}\left(\mathbb{S}^{3}\right)$, we easily verify

Lemma 3.12. Let $\overline{\mathscr{S}}_{0}$ denote the orthogonal projection of $L^{2}\left(\mathbb{S}^{3}\right)$ onto the restrictions of antiholomorphic functions. Then

$$
\overline{\mathscr{Z}} \mathscr{Q}_{0} \mathscr{Z}=\overline{\mathscr{S}}_{0}-\text { Id } \text {. }
$$

Using (3.10) and (3.13) we can further simplify the form of $\mathscr{A}^{[2]}$ :

$$
\mathscr{A}^{[2]}=2 \mathscr{S}_{0} \overline{\mathscr{Z}} \bar{\phi} \overline{\mathscr{S}}_{0} \phi \mathscr{Z} \mathscr{S}_{0} \text {. }
$$


A final simplification in the study of $\mathscr{A}^{[2]}$ follows from the observation that $\overline{\mathscr{S}}_{0}$ is a selfadjoint projection. Therefore $\overline{\mathscr{S}}_{0}=\overline{\mathscr{S}}_{0} \overline{\mathscr{S}}_{0}$ and:

$$
\mathscr{A}^{[2]}=-2 \mathscr{C}^{*} \mathscr{C} \text { where } \mathscr{C}=\overline{\mathscr{S}}_{0} \phi \mathscr{Z} \mathscr{S}_{0} .
$$

From (3.15) it is immediate that $\mathscr{A}^{[2]}$ has infinite rank if and only if $\mathscr{C}$ does.

\section{NONEMBEDDABILITY IS GENERIC}

In this section we investigate the dependence of $\mathscr{E}$ on the Fourier components of $\phi$ and the continuity properties of the map

$$
C(\phi): \phi \longrightarrow \overline{\mathscr{S}}_{0} \phi \mathscr{Z} \mathscr{S}_{0} \text {. }
$$

As generic nonembeddability follows from the Baire category theorem, it is useful to have flexibility in the choice of topology for the functional parameter, $\phi$. We show that, under rather weak hypotheses on this topology, $C(\phi)$ is generically an operator of infinite rank.

Let $\mathscr{H}$ denote the boundary values of $L^{2}$-holomorphic functions in the unit ball. The operator $C(\phi)$ is essentially a map from $\mathscr{H}$ to $\mathscr{H}$.

Let $w$ and $z$ denote complex coordinates for $\mathbb{C}^{2}$. We define the spaces of polynomials $P_{p, q}$ by

$$
P_{p, q}=\operatorname{sp}\left\{z^{a} w^{b} \bar{z}^{c} \bar{w}^{d} ; a+b=p, c+d=q\right\} .
$$

A simple calculation shows that:

$$
\int_{\mathbb{S}^{3}} z^{a} w^{b} \bar{z}^{c} \bar{w}^{d} \overline{z^{e} w^{f} \bar{z}^{g} \bar{w}^{h}} \mathrm{dVol}=\delta_{(a+g)(c+e)} \delta_{(b+h)(d+f)} B(a+g+1, b+h+1) .
$$

Here $\delta_{a b}$ is the Kronecker delta and $B(x, y)$ is the beta function:

$$
B(x+1, y+1)=\int_{0}^{1}(1-t)^{x} t^{y} d t=\frac{\Gamma(x+1) \Gamma(y+1)}{\Gamma(x+y+2)} .
$$

We set $m_{a b c d}=z^{a} w^{b} \bar{z}^{c} \bar{w}^{d} /(B(a+c+1, b+d+1))^{\frac{1}{2}}$.

As there are relations, the collection of all such monomials does not form a basis for $L^{2}\left(\mathbb{S}^{3}\right)$. A natural basis is provided by the harmonic polynomials:

$$
\begin{aligned}
H_{p, q} & =\left\{f \in P_{p, q} ; \Delta_{\mathbb{R}^{4}} f=0\right\} \\
& =\left\{f \in P_{p, q} ; \Delta_{\mathbb{S}^{3}} f=(p+q)(p+q+2) f\right\} .
\end{aligned}
$$

Let

$$
h_{j}^{0 p}=m_{00(p-j) j} ; \quad j=0, \ldots, p .
$$

This is an orthonormal basis for $H_{0, p}$ and

$$
h_{j}^{k p-k}=\overline{\mathscr{Z}}^{k} h_{j}^{0 p} /\left\|\overline{\mathscr{Z}}^{k} h_{j}^{0 p}\right\|, \quad k \leq p, 0 \leq j \leq p
$$

is an orthonormal basis for $H_{k, p-k}$. 
If $\phi \in L^{2}\left(\mathbb{S}^{3}\right)$ then it has an expansion in terms of harmonic polynomials

$$
\phi \sim \sum_{p, q=0}^{\infty} \sum_{l=0}^{p+q} b_{p q}^{l} h_{l}^{p q} .
$$

The following is a standard fact from harmonic analysis:

Lemma 4.10. If $\phi$ has $2 k \quad L^{2}$-derivatives then the coefficients in (4.9) satisfy:

$$
\left|b_{p q}^{l}\right| \leq K\|\phi\|_{H^{2 k}\left(\mathbb{S}^{3}\right)} /(p+q+1)^{2 k} .
$$

Here and in the sequel $H^{l}\left(\mathbb{S}^{3}\right), l \in \mathbb{R}$, are $L^{2}$-Sc olev spaces. The $H^{l}$ Sobolev norm is given by

$$
\|\phi\|_{H^{l}}^{2}=\sum_{p, q=0}^{\infty}\left\|\phi_{p q}\right\|_{L^{2}}^{2}(p+q+1)^{2 l} .
$$

The spherical harmonic subspaces, $H_{p, q}$, are irreducible representations of $S U(2)$. This allows us to apply elementary representation theory to study the dependence of $C(\phi)$ on the Fourier coefficients of $\phi$. We will let

It is clear that

$$
H_{p, q} \cdot H_{r, s}=\left\{f \cdot g ; f \in H_{p, q}, g \in H_{r, s}\right\} .
$$

$$
H_{p, q} \cdot H_{r, s} \subset P_{p+r, q+s}
$$

As a representation of $S U(2)$,

$$
H_{p, q} \cdot H_{r, s} \subset H_{p, q} \otimes H_{r, s} .
$$

The inequivalent, irreducible representations of $S U(2)$ are parametrized by the nonnegative integers; let $\left\{V_{n} ; n \in \mathbb{N}_{0}\right\}$ denote the list such that $\operatorname{dim} V_{n}=n+1$. It is a classical result that $H_{p, q} \cong V_{p+q}$. The Clebsch-Gordan formula for $S U(2)$ reads

$$
V_{m} \otimes V_{n} \cong V_{m+n} \oplus \cdots \oplus V_{m-n} \quad \text { for } m \geq n .
$$

On the other hand the spherical harmonic decomposition of $P_{p, q}$ is given by

$$
\begin{aligned}
& P_{p, q} \cong H_{p, q} \oplus \cdots \oplus H_{p-q, 0}, \quad p \geq q, \\
& P_{p, q} \cong H_{p, q} \oplus \cdots \oplus H_{0, q-p}, \quad q \geq p .
\end{aligned}
$$

By combining (4.12)-(4.15) we easily obtain

Lemma 4.16. Let $p, q, r$, and $s$ be nonnegative integers. If $r+s \geq p+q$ then

$$
\begin{array}{lll} 
& H_{p, q} \cdot H_{r, s} \subset H_{p+r, q+s} \oplus \cdots \oplus H_{0, q+s-p-r}, \quad \text { for } r \leq q, \\
\text { (4.17) } \quad & H_{p, q} \cdot H_{r, s} \subset H_{p+r, q+s} \oplus \cdots \oplus H_{p+r-q-s, 0}, \quad \text { for } s \leq p, \\
& H_{p, q} \cdot H_{r, s} \subset H_{p+r, q+s} \oplus \cdots \oplus H_{r-q, s-p}, \quad \text { for } r \geq q, s \geq p . \\
\text { If } r+s \leq & p+q \text { then } \\
& H_{p, q} \cdot H_{r, s} \subset H_{p+r, q+s} \oplus \cdots \oplus H_{0, q+s-p-r}, \quad \text { for } p \leq s, \\
\text { (4.18) } \quad & H_{p, q} \cdot H_{r, s} \subset H_{p+r, q+s} \oplus \cdots \oplus H_{p+r-q-s, 0}, \quad \text { for } q \leq r, \\
& H_{p, q} \cdot H_{r, s} \subset H_{p+r, q+s} \oplus \cdots \oplus H_{p-s, q-r}, \quad \text { for } r \leq q, s \leq p .
\end{array}
$$


A simple consequence of (4.17)-(4.18) is

Lemma 4.19. If $\phi$ is orthogonal to

$$
N_{0 e}=\bigoplus_{q=0}^{\infty}\left(H_{0, q} \oplus H_{1, q}\right)
$$

then $C(\phi)=0$.

Proof. Recall that the operator $\mathscr{Z}$ carries the representation $H_{p, q}$ onto $H_{p-1, q+1}$. If $p-1$ is nonnegative then the map is an isomorphism. In light of this, it suffices to show that if $p \geq 2$, then $H_{r, 1} \cdot H_{p, q}$ has a trivial projection onto $\overline{\mathscr{H}}$. This will obviously be the case if $r+p>q+1$. If $r+p \leq q+1$ and $p \geq 2$ then $r+1<p+q$. Applying (4.18) we obtain

$$
H_{r, 1} \cdot H_{p, q} \subset H_{p+r, q+1} \oplus \cdots \oplus H_{p-1, q-r}
$$

As $p \geq 2$ the assertion of the lemma follows from (4.21).

We next show that

$$
C(\phi)=0 \Longrightarrow \phi_{0 q}=\phi_{1 q}=0, \quad \forall q \in \mathbb{N}_{0} .
$$

Here and in the sequel, $f_{p q}$ denotes the orthogonal projection of $f$ into $H_{p, q}$. Note that $H_{p, 1} \cdot H_{0, q}$ and $H_{p, 1} \cdot H_{1, q}$ have nonzero projections onto $\overline{\mathscr{H}}$ only if $p \leq q$. In this case the projections are as follows

$$
\overline{\mathscr{S}}_{0}\left(H_{p, 1} \cdot H_{0, q}\right) \subset H_{0, q+1-p}, \quad \overline{\mathscr{S}}_{0}\left(H_{p, 1} \cdot H_{1, q}\right) \subset H_{0, q-p} .
$$

First suppose that $\phi_{1 q} \neq 0$, for some $q$. $\mathscr{Z}$ defines an isomorphism from $H_{q+1,0}$ onto $H_{q, 1}$ and $H_{q, 1}=\overline{H_{1, q}}$. Thus we can find an $f \in H_{q+1,0}$ such that

$$
\mathscr{Z} f=\overline{\phi_{1 q}} \text {. }
$$

It follows from (4.23) that the component of $C(\phi) f$ in $H_{0,0}$ is equal to

$$
\overline{\mathscr{S}}_{0}\left(\phi_{1 q} \mathscr{Z} f\right)=\overline{\mathscr{S}}_{0}\left(\phi_{1 q} \overline{\phi_{1 q}}\right) \neq 0 \text {. }
$$

If $C(\phi)=0$ then $\phi_{1 q}=0$ for all $q$.

Assume that $\phi \in N_{0 e}, C(\phi)=0$ but $\phi \neq 0$. The above implies that there must be a $q$ such that $\phi_{0 q} \neq 0$. We will show that there is a function, $f \in H_{q, 1}$, such that $\overline{\mathscr{S}}_{0}\left(\phi_{0 q} f\right)$ is nonzero. This leads to a contradiction.

This is a consequence of the fact that

$$
H_{q, 1} \cdot H_{1,0} \supset H_{q, 0} .
$$

The containment in (4.26) is as representations of $S U(2) . H_{q, 0}$ is an irreducible representation. Thus, to prove (4.26), it suffices to find an $f \in H_{q, 1}$ and a $g \in H_{1,0}$ such that $f \cdot g$ has a nontrivial projection into $H_{q, 0}$. If we let $f=\bar{w} z^{q}$ and $g=w$ then $f \cdot g=|w|^{2} z^{q}$. This clearly has a nontrivial projection onto $z^{q} \in H_{q, 0}$. 

that

From (4.26) it follows that we can find an $f \in H_{q+1,0}$ and a $g \in H_{1,0}$ such

$$
\overline{\mathscr{S}}_{0}(\overline{\mathscr{Z} f \cdot g})=\phi_{0 q} .
$$

From (4.27) we conclude that $\phi_{0 q} \cdot \mathscr{Z} f$ has a nontrivial projection onto $\bar{g}$. Since $\phi_{1 p}=0, p \in \mathbb{N}_{0}$, this implies that $C(\phi) f \neq 0$. We have proved the following theorem:

Theorem 4.28. The linear operator $C(\phi)=0$ if and only if the orthogonal projection of $\phi$ into $N_{0 e}$ is zero.

The monomials, $m_{r s t u}$, are useful in the study of $\mathscr{C}$ as the operator

$$
P_{r s t u}=\overline{\mathscr{S}}_{0} m_{r s t u} \mathscr{Z} \mathscr{S}_{0}
$$

satisfies

$$
\begin{aligned}
& P_{r s t u} m_{m n 00}=C_{r s t u, m n}^{a b} m_{00 a b}, \\
& \text { where } a=t+1-r-m \geq 0, \quad b=u+1-n-s \geq 0,
\end{aligned}
$$

The coefficient is given by

$$
C_{r s t u, m n}^{a b}=\frac{(t+u+3)(n(t+1)-m(u+1)) B(a+m+r+1, b+n+s+1)}{(u+1)(t+1)[B(a+m+2 r, b+n+2 s) B(m+1, n+1) B(a, b)]^{\frac{1}{2}}} .
$$

Set $\phi^{\prime}=\sum \phi_{0 q}+\phi_{1 q}$. As $C(\phi)=C\left(\phi^{\prime}\right)$ we will consider only these components of $\phi$. To make use of (4.30) we need to reexpress $\phi^{\prime}$ in terms of the monomials. Using (4.6) we easily derive

$$
\begin{aligned}
h_{j}^{1 q}=\sqrt{\frac{(j+1)(q+1-j)}{q+1}} m_{01(q-j) j}-\sqrt{\frac{j(q+2-j)}{q+1}} m_{10(q+1-j)(j-1)} \\
\quad j \in\{0, \ldots, q+1\} .
\end{aligned}
$$

Rewriting the expansion for $\phi^{\prime}$ in terms of the monomials we obtain:

$$
\begin{array}{r}
\phi^{\prime}=\sum_{q=0}^{\infty} \sum_{j=0}^{q} b_{0 q}^{j} m_{00(q-j) j}+\sum_{q=0}^{\infty} \sum_{j=0}^{q+1} \sqrt{\frac{(j+1)(q+1-j)}{q+1}} b_{1 q}^{j} m_{01(q-j) j} \\
-\sqrt{\frac{j(q+2-j)}{q+1}} b_{1 q}^{j} m_{10(q+1-j)(j-1)} .
\end{array}
$$

We need an estimate to show that the constants in (4.31) are polynomially bounded in terms of $r, s, t, u$. Using the integral representation for the beta function in (4.4) and the Cauchy-Schwarz inequality, one easily shows

$$
\begin{aligned}
& B(a+r+m+1, b+n+s+1) \\
& \quad \leq[B(a+m+2 r, b+n+2 s) B(a+m+2, b+n+2)]^{\frac{1}{2}} .
\end{aligned}
$$


It follows from (4.31) and (4.34) that

$$
\left|C_{r s t u, m n}^{a b}\right| \leq(t+u+3)^{2}\left(\frac{B(a+m+2, b+n+2)}{B(m+1, n+1) B(a, b)}\right)^{\frac{1}{2}} .
$$

We again apply the Cauchy-Schwarz inequality to obtain

$$
\begin{aligned}
B(a+ & m+2, b+n+2) \\
\leq & {[B(2(a+1), 2(b+1)) B(2(m+1), 2(n+1))]^{\frac{1}{2}} . }
\end{aligned}
$$

Using Stirling's Formula we obtain that

$$
\frac{[B(2(a+1), 2(b+1)) B(2(m+1), 2(n+1))]^{\frac{1}{2}}}{B(m+1, n+1) B(a, b)} \sim K \frac{(a b)^{\frac{5}{2}}(m n)^{\frac{1}{2}}}{(a+b)^{\frac{8}{2}}(m+n)^{\frac{1}{2}}} .
$$

Here $K$ is a positive constant. By using the arithmetic-geometric mean inequality and (4.35)-(4.37) we easily prove

Lemma 4.38. The constants given in (4.31) satisfy the inequalities

$$
\left|C_{r s t u, m n}^{a b}\right| \leq K^{\prime}(t+u+3)^{4},
$$

where $K^{\prime}$ is a fuxed positive constant.

The operator $C(\phi)$ has an expansion

$$
\begin{aligned}
C(\phi)=\sum_{q=0}^{\infty} \sum_{j=0}^{q} b_{0 q}^{j} P_{00(q-j) j}+\sum_{q=0}^{\infty} \sum_{j=0}^{q+1} & \sqrt{\frac{(j+1)(q+1-j)}{q+1}} b_{1 q}^{j} P_{01(q-j) j} \\
& -\sqrt{\frac{j(q+2-j)}{q+1}} b_{1 q}^{j} P_{10(q+1-j)(j-1)} .
\end{aligned}
$$

As stated above $C(\phi)$ is essentially a map from $\mathscr{H}$ to $\overline{\mathscr{H}}$. This operator has a matrix representation relative to the orthonormal bases $\left\{m_{m n 00} ; m, n \geq 0\right\}$ and $\left\{m_{00 p q} ; p, q \geq 0\right\}$. Denote this matrix by $A_{m n}^{p q}(\phi)$ :

$$
\begin{aligned}
A_{m n}^{p q}= & b_{0(m+n+p+q-2)}^{n+q-1} C_{00(m+p-1)(n+q-1), m n}^{p q} \\
& -\sqrt{\frac{(n+q)(m+p+1)}{m+n+p+q-1}} b_{1(m+n+p+q)}^{n+q} C_{10(m+p)(n+q-1), m n}^{p q} \\
& +\sqrt{\frac{(n+q+1)(m+p)}{m+n+p+q-1}} b_{1(m+n+p+q)}^{n+q} C_{01(m+p-1)(n+q), m n}^{p q} .
\end{aligned}
$$

The following is a simple consequence of (4.11),(4.39), and (4.41)

Proposition 4.42. Suppose that $\phi$ has $2(k+1)$ derivatives in $L^{2}\left(\mathbb{S}^{3}\right)$ for a $k \geq 2$. Then the matrix coefficients in (4.41) satisfy

$$
\left|A_{m n}^{p q}\right| \leq \frac{K\|\phi\|_{H^{2(k+1)}\left(\mathbb{S}^{3}\right)}}{\left(m^{2}+n^{2}+p^{2}+q^{2}+1\right)^{k-\frac{5}{4}}} .
$$

Here $K$ is a fixed positive constant.

The continuity properties of (4.1) are a corollary of Proposition (4.42). 
Corollary 4.44. Suppose that $\mathscr{B}$ is a complete, linear metric space of functions on $\mathbb{S}^{3}$, with metric, $d_{\mathscr{B}}(\cdot, \cdot)$, such that

$$
\begin{aligned}
& \text { (1) } \mathscr{B} \subset H^{6}\left(\mathbb{S}^{3}\right) \\
& \text { (2) } d_{\mathscr{B}}(\phi, \psi) \geq K\|\phi-\psi\|_{H^{6}\left(\mathbb{S}^{3}\right)} \text {. }
\end{aligned}
$$

Then the map (4.1) is continuous from $\left(\mathscr{B}, d_{\mathscr{B}}\right)$ to compact operators on $L^{2}\left(\mathbb{S}^{3}\right)$ with the uniform topology.

Proof. That the operator is compact follows easily from (4.43). The modulus of continuity is estimated by

$$
\begin{aligned}
& \left\|C\left(\phi_{1}\right)-C\left(\phi_{2}\right)\right\|_{\text {uniform }} \\
& \quad \leq K\left\|\phi_{1}-\phi_{2}\right\|_{H^{6}} \sup _{m, n \in \mathbb{N}_{0}}\left(\sum_{p, q \geq 0} \frac{1}{\left(1+m^{2}+n^{2}+p^{2}+q^{2}\right)^{\frac{3}{2}}}\right)^{\frac{1}{2}} \\
& \quad \leq K^{\prime} d_{\mathscr{B}}\left(\phi_{1}, \phi_{2}\right) .
\end{aligned}
$$

Define the sets:

$$
R_{n}^{\mathscr{B}}=\{\phi \in \mathscr{B} ; \text { rk } C(\phi) \leq n\} .
$$

Since the set of compact operators with less than a fixed rank is a closed subset, in the uniform topology, we have as a simple corollary of (4.44)

Corollary 4.48. If $\left(\mathscr{B}, d_{\mathscr{B}}\right)$ satisfies (4.45) then the sets $R_{n}^{\mathscr{B}}$ are closed for $n \in \mathbb{N}_{0}$.

To apply the Baire Category Theorem we need to show that the sets $R_{n}^{\mathscr{B}}$ are nowhere dense. In other words, if $\phi \in R_{n}^{\mathscr{B}}$ then we can find a sequence, $\left\{\phi_{m}\right\}$, converging to $\phi$ in $\mathscr{B}$, such that $\mathrm{rk} C\left(\phi_{m}\right)>n$. As we shall see this is easily accomplished in any space $\mathscr{B}$ which contains the antiholomorphic monomials.

Define $\mathscr{P}_{M}$ and $\overline{\mathscr{P}}_{M}$ by

$$
\begin{aligned}
& \mathscr{P}_{M}=\text { orthogonal projection of } \mathscr{H} \text { onto } \mathscr{H}_{M}=\operatorname{sp}\left\{z^{a} w^{b} ; a+b \leq M\right\} \\
& \overline{\mathscr{P}}_{M}=\text { orthogonal projection of } \overline{\mathscr{H}} \text { onto } \overline{\mathscr{H}}_{M}=\operatorname{sp}\left\{\bar{z}^{a} \bar{w}^{b} ; a+b \leq M\right\} .
\end{aligned}
$$

If $C(\phi)$ has rank $n<\infty$ then, for sufficiently large $M$ and $N$, the operator

$$
C^{M N}(\phi)=\overline{\mathscr{P}}_{N} C(\phi) \mathscr{P}_{M}
$$

also has rank $n$. It clearly would suffice to construct a sequence, $\left\{\phi_{m}\right\}$, converging to $\phi$, such that $\operatorname{rk} C^{M N}\left(\phi_{m}\right)>n$. We will think of $C^{M N}(\phi)$ as an operator between the finite-dimensional vector spaces $\mathscr{H}_{M}$ and $\overline{\mathscr{H}}_{N}$.

The subspace, $\overline{\mathscr{H}}_{Q}$, is isomorphic to $\mathbb{C}^{\operatorname{dim} \overline{\mathscr{H}}_{Q}}$. Moreover the map

$$
\overline{\mathscr{H}}_{Q} \ni \psi \rightarrow C^{M N}(\psi)
$$


is linear. Hence, if we can show that, for some $\psi_{0} \in \overline{\mathscr{H}}_{Q}$, the $\operatorname{rk} C^{M N}\left(\psi_{0}\right)>n$ then this will hold on the complement of an algebraic variety in $\overline{\mathscr{H}}_{Q}$. That this is true for sufficiently large $M, N, Q$ is an immediate consequence of (4.28).

Suppose that $M, N, Q$ are fixed so that $\operatorname{rk} C^{M N}(\phi)=n$ and we can find a $\psi_{0} \in \overline{\mathscr{H}}_{Q}$ such that $\mathrm{rk} C^{M N}\left(\psi_{0}\right)>n$. It then follows from (4.44) that

$$
\mathrm{rk} C^{M N}\left(\phi+x \psi_{0}\right)=\mathrm{rk} C_{x}>n,
$$

for sufficiently large real $x$. The map $x \longrightarrow C_{x}$ is entire. If $\operatorname{rk} C_{x}>n$ for any $x$, it must also be on the complement of a discrete subset of $\mathbb{C}$. Therefore we can find a sequence, $\left\{x_{m}\right\}$, converging to 0 so that

$$
\text { rk } C_{x_{m}}>n \text {. }
$$

The above construction proves the following theorem:

Theorem 4.54. Suppose the $\left(\mathscr{B}, d_{\mathscr{B}}\right)$ satisfies (4.45) and furthermore

$$
\overline{\mathscr{H}}_{Q} \subset \mathscr{B}, \text { for every } Q \geq 0 \text {, }
$$

then each of the closed sets, $R_{n}^{\mathscr{B}}$, is nowhere dense in $\mathscr{B}$. Thus the set

$$
R_{\mathscr{B}}^{\infty}=\{\phi \in \mathscr{B}, \text { rk } C(\phi)=\infty\}
$$

is of the second category and therefore dense.

Generic nonembeddability follows from (2.1), (2.33), and (4.54):

Theorem 4.57. Suppose the $\left(\mathscr{B}, d_{\mathscr{B}}\right)$ is a space of functions on $\mathbb{S}^{3}$, satisfying (4.45) and (4.55), and such that the functions in $\mathscr{B}$ are sufficiently differentiable for the proof of (2.1) to apply. For each $\phi \in R_{\mathscr{B}}^{\infty}$ the CR-structures on $\mathbb{S}^{3}$ defined by $\overline{\mathscr{W}}^{t \phi}, t \in D_{r(\phi)}(0)$, are nonembeddable except possibly for a countable set of values of $t$. The set of nonembeddable structures is of the second category and therefore dense in $\mathscr{B}$.

The reason for the rather general formulation of (4.57) is the diversity of interesting situations. Of course $\mathscr{C}^{\infty}\left(\mathbb{S}^{3}\right)$, with its usual topology, satisfies the hypotheses of (4.57). However this space has certain defects: (1) each structure is represented with infinite multiplicity as there is an infinite-dimensional gauge group and (2) it follows from the work of Nirenberg that nonembeddability is generic in the $\mathscr{C}^{\infty}$-topology. The equation $\bar{\partial}_{b} u=0$ generically has no local solutions, [N].

In [CL] an "infinitesimal slice" is constructed. These authors have recently shown that their slice does indeed define a local slice in which each CR-structure is represented by at most a finite-dimensional variety. The slice is defined by

$$
T_{0}=\left\{\phi ; \Im\left(\mathscr{Z}^{2} \phi\right)=0\right\} .
$$

The space $T_{0}$ is the orthogonal complement, with respect to the inner product:

$$
(f, g)=\Re \int_{\mathbb{S}^{3}} f \bar{g} \mathrm{dVol},
$$


to the set

$$
G_{0}=\left\{i \overline{\mathscr{Z}}^{2} f ; f \in \mathscr{C}^{\infty}\left(\mathbb{S}^{3} ; \mathbb{R}\right)\right\} .
$$

In the next section we obtain a different $L^{2}$-complement that splits as a sum of embeddable structures and a 'normal bundle,' $N_{0 e}$, defined in (4.20). Note that $\overline{\mathscr{H}}_{Q} \subset T_{0}$ for every $Q$ and thus on subspaces of $T_{0}$ satisfying (4.55) with topologies satisfying (4.45) the generic structure is nonembeddable.

In light of (2), a particularly interesting class is $\mathscr{C}^{\omega}\left(\mathbb{S}^{3}\right)$. For if $\phi \in \mathscr{C}^{\omega}\left(\mathbb{S}^{3}\right)$ then the equation $\overline{\mathscr{W}}^{\phi} u=0$ does have local solutions. Let $\mathscr{C}_{\delta}^{\omega}\left(\mathbb{S}^{3}\right)$ denote real analytic functions on $\mathbb{S}^{3}$ which are analytic in the $\delta$-tube about $\mathbb{S}^{3}$ in the complexification of $\mathbb{S}^{3}$. We can topologize this space by using the seminorms defined by the sup-norm topology on the $(1-1 / n) \delta$-tubes. These spaces clearly satisfy (4.45) and (4.55) as do their intersections with $T_{0}$. Thus we obtain that the generic, in the sense of $(4.57)$, perturbation in $\mathscr{C}_{\delta}^{\omega}$ or $\mathscr{C}_{\delta}^{\omega} \cap T_{0}$ is nonembeddable.

\section{SMAll PERTURbations OF THE STANDARD SPHERE}

In this section we show, that for a function $\phi$ which is sufficiently small and whose Fourier coefficients satisfy certain conditions, the structure $\overline{\mathscr{W}}^{\phi}$ embeds as a small perturbation of the standard sphere. If $\phi \in \mathscr{C}^{\infty}\left(\mathbb{S}^{3}\right)$ then it has a Fourier representation:

$$
\phi=\sum_{p, q=0}^{\infty} \phi_{p q} .
$$

Here $\phi_{p q}$ is the projection of $\phi$ into $H_{p, q}$, defined in (4.5).

We will say that

$$
\phi \in E_{0} \quad \text { if } \phi_{p q}=0 \text { for } p<q+4 ; q=0, \ldots
$$

A sufficient condition for embeddability is:

Theorem 5.3. If $\phi \in E_{0}$ and the $C^{4}\left(\mathbb{S}^{3}\right)$-norm of $\phi$ is sufficiently small then the structure $\overline{\mathscr{W}}^{\phi}$ embeds in $\mathbb{C}^{2}$ as a small perturbation of the standard sphere.

Proof. To prove the theorem we find functions, $\eta$ and $\xi$, such that

$$
\overline{\mathscr{W}}^{\phi}(w+\eta)=\overline{\mathscr{W}}^{\phi}(z+\xi)=0 .
$$

As the arguments are identical we give the proof for $w+\eta$.

Rewrite (5.4) as

$$
\overline{\mathscr{Z}} \eta=-\phi \mathscr{Z}(w+\eta) .
$$

Define an iteration by setting

$$
\eta_{0}=0, \quad \overline{\mathscr{Z}} \eta_{n+1}=-\phi \mathscr{Z}\left(w+\eta_{n}\right), \quad \eta_{n+1} \in\{\text { null } \overline{\mathscr{Z}}\}^{\perp}, \quad n \geq 0 .
$$

The main point is to show that, for each $n$, the right-hand side belongs to 
range $(\overline{\mathscr{Z}})$. Thus $\eta_{n+1}$ is actually defined. To that end we prove:

Lemma 5.7. If $\phi \in E_{0}$ and the Fourier components of $f$ satisfy

$$
f_{p q}=0 \text { for } p<q \text {, }
$$

then $\phi \mathscr{Z} f \in \operatorname{range}(\overline{\mathscr{Z}})$. The unique solution, $u$, of

$$
\overline{\mathscr{Z}} u=-\phi \mathscr{Z} f \text {, }
$$

orthogonal to null $\overline{\mathscr{Z}}$, also satisfies $u_{p q}=0, p<q$.

Proof. Let $g=\mathscr{Z} f$. The hypotheses of the lemma imply that

$$
g_{p 0}=0, \quad p=0, \ldots, \quad \text { and } g_{p q}=0 \text { for } p<q-2, q \geq 3 .
$$

It is clear that $H_{r, s} \cdot H_{p, q} \subset P_{p+r, q+s}$. If $r \geq s+4$ and $q=0,1$ or $p \geq q-2$ then $p+r>q+s$. Therefore, it follows from the spherical harmonic decomposition of $P_{p+r, q+s}$ that

$$
H_{r, s} \cdot H_{p, q} \subset H_{p+r, q+s} \oplus \cdots \oplus H_{p+r-q-s, 0} .
$$

The constraints on $p, q, r, s$ imply that $p+r-q-s \geq 2$. A moment's thought shows that the terms on the left-hand side of (5.11) are precisely the types of terms that appear in the computation of $g \cdot \phi$.

Thus we see that $g \cdot \phi$ is in the range of $\bar{Z}$. Since the $(p, q)$-Fourier coefficients of $g \cdot \phi$ vanish if $p-q<2$, it is immediate that the Fourier coefficients of the solution $u$ of (5.9) vanish if $p-q<0$. This is the assertion of the Lemma.

It is a consequence of (5.7) that the successive iterates required in (5.6) are well defined. The inverse of $\overline{\mathscr{Z}}$ on the orthogonal complement of null $\mathscr{Z}$ is the operator $-\mathscr{Q}_{0} \mathscr{Z}$. This is a restatement of (3.13). It allows us to replace the iteration in (5.6) by

$$
\eta_{0}=0, \quad \eta_{n+1}=\mathscr{Q}_{0} \mathscr{Z} \phi \mathscr{Z}\left(w+\eta_{n}\right) .
$$

In this form it is very easy to obtain the contraction property.

The operator $\mathscr{Q}_{0} \in S_{\mathscr{V}}^{-2}\left(\mathbb{S}^{3}\right)$ and $\mathscr{Z} \phi \mathscr{Z} \in S_{\mathscr{V}}^{2}\left(\mathbb{S}^{3}\right)$. Hence the composition $\mathscr{Q}_{0} \mathscr{Z} \phi \mathscr{Z} \in S_{\mathscr{V}}^{0}\left(\mathbb{S}^{3}\right)$. In particular this composition is a bounded operator on $H^{s}\left(\mathbb{S}^{3}\right)$ for any $s$. We will estimate its norm on $L^{2}$ as follows

$$
\begin{aligned}
\left\|\mathscr{Q}_{0} \mathscr{Z} \phi \mathscr{Z}\right\| & =\left\|\overline{\mathscr{Z}} \bar{\phi} \overline{\mathscr{Z}} \mathscr{Q}_{0}\right\| \\
& \leq\left\|\bar{\phi} \overline{\mathscr{Z}}^{2} \mathscr{Q}_{0}\right\|+\left\|(\overline{\mathscr{Z}} \bar{\phi}) \overline{\mathscr{Z}} \mathscr{Q}_{0}\right\| \\
& \leq|\phi|_{C^{1}\left(\mathbf{S}^{3}\right)}\left(\left\|\overline{\mathscr{X}}^{2} \mathscr{Q}_{0}\right\|+\left\|\overline{\mathscr{Z}} \mathscr{Q}_{0}\right\|\right) .
\end{aligned}
$$

As $\overline{\mathscr{Z}}^{2} \mathscr{Q}_{0} \in S_{\mathscr{V}}^{0}$ and $\overline{\mathscr{Z}} \mathscr{Q}_{0} \in S_{\mathscr{V}}^{-1}$ it follows that

$$
\left\|\mathscr{Q}_{0} \mathscr{Z} \phi \mathscr{Z}\right\| \leq C_{0}|\phi|_{C^{1}\left(\mathbf{S}^{3}\right)}
$$

for $C_{0}$ a universal constant. 
If $|\phi|_{C^{1}\left(\mathbb{S}^{3}\right)}<C_{0}^{-1}$ then the operator appearing in (5.12) defines a contraction on $L^{2}\left(\mathbb{S}^{3}\right)$. There is a unique function $\eta$ such that

$$
\eta=\left(\operatorname{Id}-\mathscr{Q}_{0} \mathscr{Z} \phi \mathscr{Z}\right)^{-1} \mathscr{Q}_{0} \mathscr{Z} \phi \mathscr{Z}(w) \text {. }
$$

The operator $\left(\mathrm{Id}-\mathscr{Q}_{0} \mathscr{Z} \phi \mathscr{Z}\right)$ belongs to $S_{\frac{1}{2}, \frac{1}{2}}^{0}\left(\mathbb{S}^{3}\right)$. A simple calculation shows that if $\|\phi\|_{L^{\infty}\left(\mathbb{S}^{3}\right)}$ is sufficiently small then its principal symbol is nonvanishing and, therefore, the operator is elliptic.

Let $A=\mathscr{Q}_{0} \mathscr{Z} \phi \mathscr{Z}$. Since Id $-A$ is invertible on $L^{2}$ it follows that there is a constant, $K$, so that

$$
\|u\|_{L^{2}} \leq K\|f\|_{L^{2}} \text { where } f=(\operatorname{Id}-A) u .
$$

We will show that, for every $s \in \mathbb{R}^{+}$, there is a constant, $K_{s}$, so that

$$
\|u\|_{H^{s}} \leq K_{s}\|f\|_{H^{s}}, \quad s \in \mathbb{R}^{+} .
$$

Let $E$ be a classical, elliptic pseudodifferential operator of order one half. The standard composition formula is valid for the composition of a classical operator and an operator of type $\left(\frac{1}{2}, \frac{1}{2}\right)$. Thus $[(\operatorname{Id}-A), E]$ is of order zero and more generally $\left[(\mathrm{Id}-A), E^{m}\right]$ is of order $\frac{1}{2}(m-1)$.

Suppose that $u \in \mathscr{C}^{\infty}$, then we have that $f \in \mathscr{C}^{\infty}$ and

$$
(\mathrm{Id}-A) E u=E f+[(\operatorname{Id}-A), E] u \text {. }
$$

Applying (5.16) to (5.18) and the fact that the commutator is of order zero we obtain that

$$
\|E u\|_{L^{2}} \leq K\left(\|E f\|_{L^{2}}+\|u\|_{L^{2}}\right) .
$$

The estimate in (5.17), for $s=\frac{1}{2}$, follows easily from (5.16), (5.19), and standard facts about Sobolev spaces. A simple induction completes the proof for $s=k / 2$ and $u \in \mathscr{C}^{\infty}$.

To obtain (5.17), for arbitrary u, we need to use a Friedrich's mollifier, $N_{\delta}, \delta \in(0,1)$. This is a family of smoothing operators with the following properties:

$$
N_{\delta} \in S^{-\infty}\left(\mathbb{S}^{3}\right), \quad \delta>0,
$$

$N_{\delta}$ is uniformly bounded in $S^{0}\left(\mathbb{S}^{3}\right)$,

$\lim _{\delta \rightarrow 0} N_{\delta}=$ Id, the limit is in the strong operator topology.

For each $\delta>0$ we have the equation

$$
(\mathrm{Id}-A) N_{\delta} u=N_{\delta} f+\left[A, N_{\delta}\right] u .
$$

It follows from (5.20) that the commutator $\left[A, N_{\delta}\right]$ is uniformly bounded in $S_{\frac{1}{2}, \frac{1}{2}}^{-\frac{1}{2}}\left(\mathbb{S}^{3}\right)$ and converges to zero in the strong operator topology. Let $\delta$ and $\delta^{\prime}$ be two positive numbers. We can apply (5.17) to (5.21) to conclude that

$$
\left\|N_{\delta} u-N_{\delta^{\prime}} u\right\|_{H^{s}} \leq K_{s}\left(\left\|N_{\delta} f-N_{\delta^{\prime}} f\right\|_{H^{s}}+\left\|\left[A, N_{\delta}\right] u-\left[A, N_{\delta^{\prime}}\right] u\right\|_{H^{s}}\right) .
$$


Suppose that $f \in H^{\frac{k}{2}}$ for some positive $k$. As $(\operatorname{Id}-A)$ is invertible on $L^{2}$, we can assume that $\lim _{\delta \rightarrow 0} N_{\delta} u=u$ in $L^{2}$. Using this as a starting point and (5.22) to bootstrap, we can show that $N_{\delta} u$ converges to $u$ in $H^{\frac{k}{2}}$. From this we conclude that for, $f \in H^{\frac{k}{2}}$, there is a unique $u \in H^{\frac{k}{2}}$ satisfying $(\operatorname{Id}-A) u=f$ and (5.17), with $s=\frac{k}{2}$. The result for general $s>0$ follows by interpolation.

Thus elliptic regularity along with $(5.15)$ imply that $\eta \in \mathscr{C}^{\infty}\left(\mathbb{S}^{3}\right)$. The same is true of course for $\xi$. It follows, from the Sobolev embedding theorem, that the $C^{1}\left(\mathbb{S}^{3}\right)$-norm is estimated by the $H^{3}\left(\mathbb{S}^{3}\right)$-norm. Therefore (5.15) and (5.17) imply that such an estimate follows from a bound on the $C^{4}\left(\mathbb{S}^{3}\right)$-norm of $\phi$.

Given $\varepsilon>0$, we can find a $\delta>0$, such that if $|\phi|_{C^{4}\left(\mathcal{S}^{3}\right)}<\delta$ then the $C^{1}$ norms of $\eta$ and $\xi$ are less than $\varepsilon$. The Jacobian determinant of the map,

$$
(w, z) \longrightarrow(w+\eta(w, z), z+\xi(w, z)),
$$

will be positive if $\varepsilon$ is small enough. Taking $\varepsilon$ even smaller, we can ensure that the map is globally injective. This completes the proof of the theorem.

As stated in $\S 4$ the linear space $E_{0} \oplus N_{0 e}$ defines a complement to $G_{0}$. This is not quite true, we need to discard a piece of $E_{0}$. Let

$$
E_{1}=\left\{\phi ; \phi_{p q}=0 \text { for } p<q+5\right\}
$$

and let

$$
E_{2}=\left\{\phi \in \oplus_{q=0}^{\infty} H_{q+4, q} ; \Im \mathscr{Z}^{2} \phi=0\right\} .
$$

The space $T_{0}^{\prime}=N_{0 e} \oplus E_{1} \oplus E_{2}$ does define a complement to $G_{0}$. This is because every solution of $\Im \mathscr{Z}^{2} \phi=0$ has a unique decomposition as

$$
\phi=\phi_{1}+\phi_{2}+\phi_{-}+\phi_{+} \text {. }
$$

The terms $\phi_{1} \in N_{0 e}, \phi_{2} \in E_{2}$, and $\phi_{-} \in E_{1}$. The remaining factor belongs to the space

$$
E_{3}=\bigoplus_{p=4}^{\infty} \bigoplus_{q=p-3}^{\infty} H_{p, q} .
$$

The map from $\phi_{-}$to $\phi_{+}$is bounded and boundedly invertible on $L^{2}\left(\mathbb{S}^{3}\right)$. A moment's thought shows that

$$
E_{1} \oplus E_{2} \oplus N_{0 e} \bigcap G_{0}=\{0\} .
$$

In fact the angle between these spaces is bounded away from zero. It is clear that $T_{0}^{\prime}$ defines a complement to $G_{0}$ and an infinitesimal slice linearly equivalent to $T_{0}$.

The main outstanding problem is the relationship between the topologies on embeddable CR-structures defined by the parametrization $(0.3)$ and those induced by the embeddings themselves. Let $N \subset \mathbb{C}^{n}$ be a strictly pseudoconvex hypersurface and $\mathscr{B}_{N}$ the set of $\mathscr{C}^{\infty}$-biholomorphic maps defined on $N$. Let $\operatorname{Diff}_{c}\left(N_{1}, N_{2}\right)$ denote the set of 'compactly supported' diffeomorphisms of $\mathbb{C}^{n}$ 
that carry $N_{1}$ onto $N_{2}$. We define the $C^{2}$-embedded distance from $N_{2}$ to the biholomorphic equivalence class of $N_{1}$ by

$$
R_{N_{2}}\left(\left[N_{1}\right]\right)=\inf _{F \in \mathscr{B}_{N_{1}}} \sup _{\Psi \in \operatorname{Diff}_{c}\left(F\left(N_{1}\right), N_{2}\right)}\|\Psi-\operatorname{Id}\|_{C^{2}}+\left\|\Psi^{-1}-\mathrm{Id}\right\|_{C^{2}}
$$

Using an argument of Rosay, [R], one can show that these functions seperate biholomorphic equivalence classes. This was essentially proved in [GK]. There it is also shown that, replacing $C^{2}$ by $C^{1-\varepsilon}$, one cannot seperate points. By using more than two derivatives; one can also define metric topologies.

Let $\phi=\phi_{0}+\phi^{\sharp} ; \phi_{0} \in E_{1}+E_{2}, \phi^{\sharp} \in N_{0 e}$. From Theorem (5.3) it follows that the CR-structure defined by $\phi_{0}$ is realizable as a small perturbation of $\mathbb{S}^{3}$. The ' $H^{s}$-embedded distance' to the unit sphere is $O\left(\left\|\phi_{0}\right\|_{H^{s}}\right)$. We show that, for sufficiently small $\phi_{0}, \phi^{\sharp} \neq 0$, this is not the case for the structure defined by $\phi$.

Let $w^{\prime}=w+\eta, z^{\prime}=z+\xi$ be the solutions of $\overline{\mathscr{W}}^{\phi_{0}} f=0$ defined by (5.6).

Theorem 5.25. There exist positive constants $C_{1}, C_{2}, s_{0}$ such that given $K>0$ if

$$
\left\|\phi_{0}\right\|_{H^{s_{0}}} \leq C_{1}, \quad\left\|\phi^{\sharp}\right\|_{H^{\frac{13}{2}}} \leq C_{2} / K, \quad \phi^{\sharp} \neq 0
$$

then at least one of the equations

$$
\overline{\mathscr{W}}^{\phi}\left(w^{\prime}+\tilde{\eta}\right)=0, \quad \overline{\mathscr{W}}^{\phi}\left(z^{\prime}+\tilde{\xi}\right)=0,
$$

does not have a solution with

$$
\|\tilde{\eta}\|_{C^{1}} \leq K\left\|\phi^{\sharp}\right\|_{C^{1}}, \quad\|\tilde{\xi}\|_{C^{1}} \leq K\left\|\phi^{\sharp}\right\|_{C^{1}} .
$$

Remarks. The examples in $[\mathrm{GK}]$ indicate that the estimates in (5.28) represent some sort of borderline. It would be very interesting to know if the functions, defined in (5.24) with $C^{2}$ replaced by $C^{1}$, still seperate biholomorphic equivalence classes.

The estimates, (5.28), can be replaced by any estimates of the form:

$$
\|\tilde{\eta}\|_{C^{1}} \leq K\left\|\phi^{\sharp}\right\|_{\mathscr{B}}, \quad\|\tilde{\xi}\|_{C^{1}} \leq K\left\|\phi^{\sharp}\right\|_{\mathscr{B}} .
$$

All that the proof requires is, for some $s, C \in \mathbb{R}$,

$$
\|f\|_{\mathscr{B}} \leq C\|f\|_{H^{s}}
$$

The estimate on $\phi^{\sharp}$ in (5.26) is then replaced by an estimate of the form

$$
\left\|\phi^{\sharp}\right\|_{H^{s^{\prime}}} \leq C_{2} / K
$$

for some $s^{\prime} \in \mathbb{R}$.

Proof. Let $\overline{\mathscr{W}}=\overline{\mathscr{W}}^{\phi_{0}}$; rewrite $(5.27)$ as

$$
\overline{\mathscr{W}} \tilde{\eta}=-\phi^{\sharp} \mathscr{Z}\left(w^{\prime}+\tilde{\eta}\right), \quad \overline{\mathscr{W}} \widetilde{\xi}=-\phi^{\sharp} \mathscr{Z}\left(z^{\prime}+\tilde{\xi}\right) .
$$


We show that these equations are not both solvable. One of the right-hand sides does not lie in

$$
\text { range } \overline{\mathscr{W}}=\left(\text { null } \overline{\mathscr{W}}^{*}\right)^{\perp} \text {. }
$$

The argument is by contradiction.

A consequence of Theorem (5.3) is that range $\overline{\mathscr{W}}$ is closed. Let $\mathscr{Q}$ denote the partial inverse of $\square_{b}^{\phi_{0}}$ and $\overline{\mathscr{S}}$ the orthogonal projection onto the (range $\left.\overline{\mathscr{W}}\right)^{\perp}$. One easily establishes

$$
\overline{\mathscr{S}} \overline{\mathscr{W}}=0 \text { and } \overline{\mathscr{W}} \mathscr{Q} \overline{\mathscr{W}}^{*}=\mathrm{Id}-\overline{\mathscr{S}} \text {. }
$$

We assume that

$$
\|\tilde{\eta}\|_{C^{1}} \leq K\left\|\phi^{\sharp}\right\|_{C^{1}} \text { and }\|\tilde{\xi}\|_{C^{1}} \leq K\left\|\phi^{\sharp}\right\|_{C^{1}} \text {. }
$$

We will derive a contradiction by showing that $\overline{\mathscr{S}}$ applied to either $\phi^{\sharp} \mathscr{Z}(\eta+\tilde{\eta}+w)$ or $\phi^{\sharp} \mathscr{Z}(\xi+\widetilde{\xi}+z)$ is nonzero. First we observe that

$$
\begin{aligned}
\left\|\overline{\mathscr{S}} \phi^{\sharp} \mathscr{Z} \tilde{\eta}\right\|_{L^{2}} & \leq\left\|\phi^{\sharp} \mathscr{Z} \tilde{\eta}\right\|_{L^{2}} \\
& \leq C\|\mathscr{Z} \tilde{\eta}\|_{L^{\infty}}\left\|\phi^{\sharp}\right\|_{L^{2}} .
\end{aligned}
$$

The Sobolev embedding theorem implies that

$$
\left\|\phi^{\sharp}\right\|_{C^{1}} \leq C\left\|\phi^{\sharp}\right\|_{H^{3}} \text {. }
$$

From (5.30), (5.31), (5.32), and the fact that

$$
\|u\|_{H^{s}} \leq\left(\|u\|_{H^{s+t}}\|u\|_{H^{s-t}}\right)^{\frac{1}{2}}
$$

we deduce that

$$
\|\overline{\mathscr{S}} \phi \mathscr{Z} \tilde{\eta}\|_{L^{2}} \leq C^{\prime} K\left\|\phi^{\sharp}\right\|_{H^{\frac{13}{2}}}\left\|\phi^{\sharp}\right\|_{H^{-\frac{1}{2}}},
$$

for some constant $C^{\prime}$.

On the other hand, we will show that there is a constant, $C_{0}$, such that either

$$
\left\|\overline{\mathscr{S}} \phi^{\sharp} \mathscr{Z}(w+\eta)\right\|_{L^{2}} \geq C_{0}\left\|\phi^{\sharp}\right\|_{H^{-\frac{1}{2}}} \text { or }\left\|\overline{\mathscr{S}} \phi^{\sharp} \mathscr{Z}(z+\xi)\right\|_{L^{2}} \geq C_{0}\left\|\phi^{\sharp}\right\|_{H^{-\frac{1}{2}}} \text {. }
$$

As a first step, we prove the following inequality

Lemma 5.35. Let $\phi_{i}^{\sharp}, i=0,1$ denote the projections of $\phi^{\sharp}$ into $\oplus H_{i, q}, i=$ 0,1 . There exists a positive constant $C^{\prime}$ such that

$$
\left\|\overline{\mathscr{S}}_{0} \phi^{\sharp} \mathscr{Z} z\right\|_{L^{2}}^{2}+\left\|\overline{\mathscr{S}}_{0} \phi^{\sharp} \mathscr{Z} w\right\|_{L^{2}}^{2} \geq C^{\prime}\left(\left\|\phi_{0}^{\sharp}\right\|_{L^{2}}^{2}+\left\|\phi_{1}^{\sharp}\right\|_{H^{-\frac{1}{2}}}^{2}\right) \text {. }
$$

Proof. It is obvious that $\bar{z} \phi_{0}^{\sharp}$ and $\bar{w} \phi_{0}^{\sharp}$ also lie in $\oplus H_{0, q}$. Let

$$
\bar{z} \phi_{1}^{\sharp}=a_{0}+a_{1} \quad \text { and } \quad \bar{w} \phi_{1}^{\sharp}=b_{0}+b_{1}
$$

be the decompositions into $\oplus H_{0, q}$ and $\oplus H_{1, q}$. Then

$$
\overline{\mathscr{S}}_{0} \phi^{\sharp} \mathfrak{Z} w=-\bar{z} \phi_{0}^{\sharp}-a_{0}, \quad \overline{\mathscr{S}}_{0} \phi^{\sharp} \mathscr{Z} z=\bar{w} \phi_{0}^{\sharp}+b_{0} .
$$


Observe that

$$
\begin{gathered}
\bar{w} \phi_{0}^{\sharp}, \quad \bar{z} \phi_{0}^{\sharp} \in \oplus H_{0, q} ; \quad a_{1}, b_{1} \oplus H_{1, q}, \\
\left\langle\bar{z} \phi_{0}^{\sharp}, a_{1}\right\rangle=\left\langle\bar{w} \phi_{0}^{\sharp}, b_{1}\right\rangle=0 .
\end{gathered}
$$

From this we conclude that

$$
\begin{aligned}
\left\langle\bar{z} \phi_{0}^{\sharp}, a_{0}\right\rangle & =\left\langle\bar{z} \phi_{0}^{\sharp}, \bar{z} \phi_{1}^{\sharp}\right\rangle, \\
\left\langle\bar{w} \phi_{0}^{\sharp}, b_{0}\right\rangle & =\left\langle\bar{w} \phi_{0}^{\sharp}, \bar{w} \phi_{1}^{\sharp}\right\rangle
\end{aligned}
$$

and, therefore,

$$
\left\|\bar{z} \phi_{0}^{\sharp}+a_{0}\right\|_{L^{2}}^{2}+\left\|\bar{w} \phi_{0}^{\sharp}+b_{0}\right\|_{L^{2}}^{2}=\left\|\phi_{0}^{\sharp}\right\|_{L^{2}}^{2}+\left\|a_{0}\right\|_{L^{2}}^{2}+\left\|b_{0}\right\|_{L^{2}}^{2} .
$$

Let $a_{1 q}$ and $b_{1 q}$ denote the components of $a_{1}$ and $b_{1}$ in $H_{1, q+1}$. Applying $\mathscr{Z}$ to the equations in (5.37) we obtain

$$
\bar{z} \mathscr{Z} \phi_{1}^{\sharp}=\mathscr{Z} a_{1}, \quad \bar{w} \mathscr{Z} \phi_{1}^{\sharp}=\mathscr{Z} b_{1} .
$$

A simple calculation then shows

$$
\left\|a_{1 q}\right\|_{L^{2}}^{2}+\left\|b_{1 q}\right\|_{L^{2}}^{2}=\left(\frac{q+1}{q+2}\right)\left\|\phi_{1 q}^{\sharp}\right\|^{2} .
$$

If we let $a_{0 q}$ and $b_{0 q}$ denote the components of $a_{0}$ and $b_{0}$ in $H_{0, q}$ then one easily shows that

$$
\left\|\phi_{1 q}^{\sharp}\right\|_{L^{2}}^{2}=\left\|a_{0 q}\right\|_{L^{2}}^{2}+\left\|a_{1 q}\right\|_{L^{2}}^{2}+\left\|b_{0 q}\right\|_{L^{2}}^{2}+\left\|b_{1 q}\right\|_{L^{2}}^{2} .
$$

It follows from $(5.40)-(5.41)$ that

$$
\left\|a_{0 q}\right\|_{L^{2}}^{2}+\left\|b_{0 q}\right\|_{L^{2}}^{2}=\left(\frac{1}{q+2}\right)\left\|\phi_{1 q}^{\sharp}\right\|_{L^{2}}^{2} .
$$

We apply Lemma (5.35) to show:

Lemma 5.43. For $\phi_{0}$ sufficiently small there is a positive constant $C^{\prime \prime}$ such that

$$
\left\|\overline{\mathscr{S}}_{0} \phi^{\sharp} \mathscr{Z}(w+\eta)\right\|_{L^{2}}^{2}+\left\|\overline{\mathscr{S}}_{0} \phi^{\sharp} \mathscr{Z}(z+\xi)\right\|_{L^{2}}^{2} \geq C^{\prime \prime}\left(\left\|\phi_{0}^{\sharp}\right\|_{L^{2}}^{2}+\left\|\phi_{1}^{\sharp}\right\|_{H^{-\frac{1}{2}}}^{2}\right) \text {. }
$$

Proof. We need to estimate $\left\|\overline{\mathscr{S}}_{0} \phi^{\sharp} \mathscr{Z} \eta\right\|_{L^{2}}$ and $\left\|\overline{\mathscr{S}}_{0} \phi^{\sharp} \mathscr{Z} \xi\right\|_{L^{2}}$. We will give the details of the argument for $\eta$. From the triangle inequality it is clear that

$$
\left\|\overline{\mathscr{S}}_{0} \phi^{\sharp} \mathscr{Z} \eta\right\|_{L^{2}} \leq\left\|\overline{\mathscr{S}}_{0} \phi_{0}^{\sharp} \mathscr{Z} \eta\right\|_{L^{2}}+\left\|\overline{\mathscr{S}}_{0} \phi_{1}^{\sharp} \mathscr{Z} \eta\right\|_{L^{2}} \text {. }
$$

The first term on the right-hand side is estimated by

$$
\left\|\overline{\mathscr{S}}_{0} \phi_{0}^{\sharp} \mathscr{Z} \eta\right\|_{L^{2}} \leq C_{1}\|\mathscr{Z} \eta\|_{L^{\infty}}\left\|\phi_{0}^{\sharp}\right\|_{L^{2}} \text {. }
$$


To estimate the second term we use

Lemma 5.48. There exists a positive constant $C_{j}^{\prime}$ such that if $f \in \mathscr{C}^{\infty}\left(\mathbb{S}^{3}\right)$ and $\psi \in \oplus_{q=0}^{\infty} H_{j, q}, \quad j \geq 0$

then

$$
\left\|\overline{\mathscr{S}}_{0}(\psi f)\right\|_{L^{2}} \leq C_{j}^{\prime}\left\|\overline{\mathscr{Z}}^{j} f\right\|_{L^{\infty}}\|\psi\|_{H^{\frac{-j}{2}}} \text {. }
$$

Proof. It is an elementary exercise, using spherical harmonics, to show that there exists a unique $\tilde{\psi} \in \oplus H_{0, q}$ such that

$$
\overline{\mathscr{Z}}^{j} \tilde{\psi}=\psi, \quad \widetilde{\psi} \perp \overline{\mathscr{Z}}^{j} .
$$

From (5.50) it follows easily that there is a constant $C_{j}^{\prime}$ such that

$$
\|\widetilde{\psi}\|_{L^{2}} \leq C_{j}^{\prime}\|\psi\|_{H^{\frac{-j}{2}}} .
$$

Since $\overline{\mathscr{S}}_{0} \overline{\mathscr{Z}}=0$ we see that

$$
\overline{\mathscr{S}}_{0}\left(f \overline{\mathscr{Z}}^{j} \widetilde{\psi}\right)=(-1)^{j} \overline{\mathscr{S}}_{0}\left(\widetilde{\psi} \overline{\mathscr{Z}}^{j} f\right) .
$$

The lemma follows from (5.51) and (5.52).

From this lemma it follows that

$$
\left\|\overline{\mathscr{S}}_{0} \phi_{1}^{\sharp} \mathscr{Z} \eta\right\|_{L^{2}} \leq C_{1}^{\prime}\|\overline{\mathscr{Z}} \mathscr{Z} \eta\|_{L^{\infty}}\left\|\phi_{1}^{\sharp}\right\|_{H^{-\frac{1}{2}}} .
$$

Combining (5.17),(5.47), and (5.53) and applying the Sobolev embedding theorem we obtain

$$
\left\|\overline{\mathscr{S}}_{0} \phi^{\sharp} \mathscr{Z} \eta\right\|_{L^{2}} \leq C\left\|\phi_{0}\right\|_{H^{4}}\left(\left\|\phi_{0}^{\sharp}\right\|_{L^{2}}+\left\|\phi_{1}^{\sharp}\right\|_{H^{-\frac{1}{2}}}\right) .
$$

Combining (5.36) and (5.54) completes the proof of (5.44).

Now we must estimate the following errors $\left\|\left(\overline{\mathscr{S}}-\overline{\mathscr{S}}_{0}\right) \phi^{\sharp} \mathscr{Z}(w+\eta)\right\|_{L^{2}}$ and $\left\|\left(\overline{\mathscr{S}}-\overline{\mathscr{S}}_{0}\right) \phi^{\sharp} \mathscr{Z}(z+\xi)\right\|_{L^{2}}$. As before we only present the argument for $\eta$. These estimates follow from

Proposition 5.55. Let $\phi \in \mathscr{C}^{\infty}\left(\mathbb{S}^{3}\right) \cap E_{0}$. For sufficiently large $l \in \mathbb{N}$, the map $\phi \longrightarrow \mathscr{S}^{\phi}$ is continuous from a ball about $0 \in C^{l}\left(\mathbb{S}^{3}\right) \cap E_{0}$ to bounded operators on $L^{2}\left(\mathbb{S}^{3}\right)$, with the uniform topology.

Proof. If the operator $\mathbf{\square}_{b}^{\psi}$ has no spectrum in the interval $(0, r], r>0$ then

$$
2 \pi i \mathscr{S}^{\phi}=-\int_{|\lambda|=r} R^{\phi}(\lambda) d \lambda, \quad \text { where } R^{\phi}(\lambda)=\left(\boldsymbol{\square}_{b}^{\phi}-\lambda\right)^{-1} .
$$

The continuity statement follows easily from (5.56), once it is established that there exist $l \in \mathbb{N}, \varepsilon>0$, and $r>0$ such that:

$$
\text { If }\|\phi\|_{C^{\prime}} \leq \varepsilon \quad \text { then } \operatorname{spec}\left(\boldsymbol{\square}_{b}^{\psi}\right) \cap(0, r]=\varnothing \text {. }
$$

Before establishing (5.57), we show how it implies the proposition. 
Let $\psi, \phi$ be two smooth functions in $E_{0}$ for which (5.57) holds. A simple calculation using $(0.5)$ shows that

$$
\begin{aligned}
& 2 \pi i\left(\mathscr{S}^{\psi}-\mathscr{S}^{\phi}\right) \\
& \quad=-\int_{|\lambda|=\frac{1}{2} r} R^{\psi}(\lambda)\left[\mathscr{Z}\left(f_{1}\right) \overline{\mathscr{Z}}+\overline{\mathscr{Z}}\left(f_{2}\right) \overline{\mathscr{Z}}+\mathscr{Z}\left(f_{3}\right) \mathscr{Z}+\overline{\mathscr{Z}}\left(f_{4}\right) \mathscr{Z}\right] R^{\phi}(\lambda) d \lambda
\end{aligned}
$$

where $f_{1}=N_{\psi}-N_{\phi}, f_{2}=\bar{\psi} N_{\psi}-\bar{\phi} N_{\phi}, f_{3}=\psi N_{\psi}-\phi N_{\phi}$,

$$
f_{4}=|\psi|^{2} N_{\psi}-|\phi|^{2} N_{\phi} \quad \text { with } N_{h}=\left(1+|h|^{2}\right) /\left(1-|h|^{2}\right)^{2} \text {. }
$$

The condition, (5.57), and the construction in $\S 2$ imply that the operators, $\overline{\mathscr{Z}} R^{\psi}(\lambda), \mathscr{Z} R^{\psi}(\lambda), \overline{\mathscr{Z}} R^{\phi}(\lambda)$, and $\mathscr{Z} R^{\phi}(\lambda)$, and their adjoints are uniformly bounded on $L^{2}$. These bounds may require taking a larger $l$ and smaller $\varepsilon$ than that obtained in (5.57). Denote the uniform bound by $M$.

Since the operators $\mathscr{S}^{\psi}$ and $\mathscr{S}^{\phi}$ are selfadjoint it follows that

$$
\left\|\mathscr{S}^{\psi}-\mathscr{S}^{\phi}\right\|=\sup _{f, g \in L^{2}}\left\langle\left(\mathscr{S}^{\psi}-\mathscr{S}^{\phi}\right) f, g\right\rangle /\|f\|\|g\| \text {. }
$$

Using (5.59) in (5.58) we obtain that

$$
\left\|\mathscr{S}^{\psi}-\mathscr{S}^{\phi}\right\| \leq C r M^{2}\|\psi-\phi\|_{L^{\infty}} .
$$

Here $C$ is a absolute constant depending only on $l$ and $\varepsilon$.

All that remains is the verification of (5.57). This follows from the estimate, (5.17), and the argument used in [Ko]. In this paper, Kohn shows that the range of $\bar{\partial}_{b}$ is closed in $L^{2}$, for the boundary of a pseudoconvex domain in $\mathbb{C}^{n}$. More precisely, he shows that for any $u \in \mathscr{C}^{\infty}(\partial D)$, there exists a function $v \in C^{1}(\partial D)$, such that

$$
\bar{\partial}_{b} u=\bar{\partial}_{b} v \text { and }\|v\|_{L^{2}} \leq K(D)\left\|\bar{\partial}_{b} u\right\|_{L^{2}} .
$$

The constant $K(D)$ depends only on the domain.

The argument is microlocal and clearly implies that the constant actually depends on only finitely many derivatives of the data defining $\partial D$. Our estimate, (5.17), implies that, if $l$ is taken sufficiently large and $\varepsilon$ sufficiently small, then the realization of the CR-structure, $\overline{\mathscr{W}}^{\psi}$, defined by (5.4) satisfies such estimates. Moreover, the hermitian structure induced from the embedding is an arbitrarily small perturbation of that defined by the standard metric on $\mathbb{S}^{3}$. From this discussion, it is clear that there exists a constant, $K(l, \varepsilon)$, such that

$$
\|u\|_{L^{2}} \leq K(l, \varepsilon)\left\|\overline{\mathscr{W}}^{\phi} u\right\|_{L^{2}} \text { for } u \in\{\overline{\mathscr{W}}\}^{\perp} \text {, if } \phi \in E_{0},\|\phi\|_{C^{\prime}} \leq \varepsilon .
$$

This estimate is equivalent to (5.57).

Henceforth we assume that

$$
\left\|\phi_{0}\right\|_{C^{\prime}} \leq \varepsilon
$$


The constants are those obtained in the proof of Proposition (5.55). Recall that $\overline{\mathscr{S}}_{u}=\overline{\mathscr{S}} \bar{u}$. Thus, the map $\phi \longrightarrow \overline{\mathscr{S}}^{\phi}$ also has the continuity property described in Proposition (5.55).

The first step in estimating the error term is to apply the triangle inequality.

$$
\begin{aligned}
\left\|\left(\overline{\mathscr{S}}-\overline{\mathscr{S}}_{0}\right) \phi^{\sharp} \mathscr{Z}(w+\eta)\right\|_{L^{2}} \leq & \left\|\left(\overline{\mathscr{S}}-\overline{\mathscr{S}}_{0}\right) \phi_{0}^{\sharp} \mathscr{Z}(w+\eta)\right\|_{L^{2}} \\
& +\left\|\left(\overline{\mathscr{S}}-\overline{\mathscr{S}}_{0}\right) \phi_{1}^{\sharp} \mathcal{Z}(w+\eta)\right\|_{L^{2}} .
\end{aligned}
$$

It follows from Proposition (5.55) that there exists a constant $C_{2}$ such that

$$
\left\|\left(\overline{\mathscr{S}}-\overline{\mathscr{S}}_{0}\right) \phi_{0}^{\sharp} \mathscr{Z}(w+\eta)\right\|_{L^{2}} \leq C_{2}\left\|\phi_{0}\right\|_{L^{\infty}}\left\|\phi_{0}^{\sharp} \mathscr{Z}(w+\eta)\right\|_{L^{2}} .
$$

In virtue of (5.17) and (5.63) it follows that there is another constant $C_{3}$ such that

$$
\|\mathscr{Z}(w+\eta)\|_{L^{\infty}} \leq C_{3} .
$$

From (5.65), (5.66) we obtain

$$
\left\|\left(\overline{\mathscr{S}}-\overline{\mathscr{S}}_{0}\right) \phi_{0}^{\sharp} \mathscr{Z}(w+\eta)\right\|_{L^{2}} \leq C_{2}^{\prime}\left\|\phi_{0}\right\|_{L^{\infty}}\left\|\phi_{0}^{\sharp}\right\|_{L^{2}} .
$$

To estimate the second term we write $\phi_{1}^{\sharp}=\overline{\mathscr{Z}} \psi$ as in (5.50) to obtain:

$$
\begin{aligned}
\|(\overline{\mathscr{S}} & \left.-\overline{\mathscr{S}}_{0}\right) \phi_{1}^{\sharp} \mathscr{Z}(w+\eta) \|_{L^{2}} \\
& =\left\|\left(\overline{\mathscr{S}}_{-} \overline{\mathscr{S}}_{0}\right)[\overline{\mathscr{Z}}(\psi \mathscr{Z}(w+\eta))-\psi \overline{\mathscr{X}} \mathscr{Z}(w+\eta)]\right\|_{L^{2}} \\
& \leq\left\|\left(\overline{\mathscr{S}}-\overline{\mathscr{S}}_{0}\right) \overline{\mathscr{Z}}(\psi \mathscr{Z}(w+\eta))\right\|_{L^{2}}+\left\|\left(\overline{\mathscr{S}}-\overline{\mathscr{S}}_{0}\right)(\psi \overline{\mathscr{Z}} \mathscr{Z}(w+\eta))\right\|_{L^{2}} .
\end{aligned}
$$

Arguing as above, the second term in (5.68) satisfies

$$
\begin{aligned}
\left\|\left(\overline{\mathscr{S}}-\overline{\mathscr{S}}_{0}\right)(\psi \overline{\mathscr{X}} \mathscr{Z}(w+\eta))\right\|_{L^{2}} & \leq C_{2}^{\prime \prime}\left\|\phi_{0}\right\|_{L^{\infty}}\|\psi\|_{L^{2}} \\
& \leq C_{2}^{\prime \prime}\left\|\phi_{0}\right\|_{L^{\infty}}\left\|\phi_{1}^{\sharp}\right\|_{H^{-\frac{1}{2}}} .
\end{aligned}
$$

To estimate the other term we observe (5.28). implies that

$$
\overline{\mathscr{S}} \overline{\mathcal{Z}}=-\overline{\mathscr{S}} \phi_{0} \mathscr{Z} \text {. }
$$

Using (5.70), $\overline{\mathscr{S}}_{0} \overline{\mathscr{X}}=0$, and the fact that $\mathscr{Z} \psi=\mathscr{Z}^{2} w=0$, we see that the first term in (5.68) can be rewritten and estimated by

$$
\begin{aligned}
\left\|\left(\overline{\mathscr{S}}-\overline{\mathscr{S}}_{0}\right) \overline{\mathscr{X}}(\psi \mathscr{Z}(w+\eta))\right\|_{L^{2}} & =\left\|\overline{\mathscr{S}} \phi_{0} \psi \mathscr{Z}^{2} \eta\right\|_{L^{2}} \\
& \leq\left\|\phi_{0} \mathscr{Z}^{2} \eta\right\|_{L^{\infty}}\left\|\phi_{1}^{\sharp}\right\|_{H^{-\frac{1}{2}}} .
\end{aligned}
$$

Combining (5.67), (5.69), and (5.71) we obtain that, for some finite constant C,

$$
\left\|\left(\overline{\mathscr{S}}-\overline{\mathscr{S}}_{0}\right) \phi^{\sharp} \mathscr{Z}(w+\eta)\right\|_{L^{2}} \leq C\left\|\phi_{0}\right\|_{L^{\infty}}\left(\left\|\phi_{0}^{\sharp}\right\|_{L^{2}}+\left\|\phi_{1}^{\sharp}\right\|_{H^{-\frac{1}{2}}}\right) .
$$

Here we assume that $\phi_{0}$ satisfies (5.63). 
The estimates (5.44) and (5.72) imply (5.34). Let us suppose that the inequality in (5.34) holds for $\eta$. Using (5.33) we have the estimate:

$$
\left\|\overline{\mathscr{S}} \phi^{\sharp} \mathscr{Z}(w+\eta+\tilde{\eta})\right\|_{L^{2}} \geq C_{0}\left\|\phi^{\sharp}\right\|_{H^{-\frac{1}{2}}}\left(1-\frac{C^{\prime} K}{C_{0}}\left\|\phi^{\sharp}\right\|_{H^{\frac{13}{2}}}\right),
$$

provided $\left\|\phi_{0}\right\|_{L^{\infty}}$ is sufficiently small. If

$$
\left\|\phi^{\sharp}\right\|_{H^{\frac{13}{2}}}<C_{0} / C^{\prime} K
$$

the right-hand side in (5.73) is positive. This contrac.cts $\left(5.27^{\prime}\right)$ and (5.29), thus completing the proof of the theorem.

Theorems (5.3) and (5.25) suggest the following local picture: Among the structures parametrized by $T_{0}^{\prime}$, the structures lying in $E_{1} \oplus E_{2}$ are embeddable in $\mathbb{C}^{2}$ and $N_{0 e}$ is the 'normal bundle' to the codimension one, embeddable structures.

If any embeddable, small perturbation of the standard structure actually embeds as a small perturbation of the standard sphere then $N_{0 e}$ would represent the normal bundle to the embeddable structures in $T_{0}^{\prime}$. The results of $\S 4$ imply that the subset of structures in $T_{0}^{\prime} \backslash E_{1} \oplus E_{2}$ which are nonembeddable is a dense $G_{\delta}$. The results of Cheng and Lee and the remarks at the end of $\S 4$ make it plausible that each CR-structure on $\mathbb{S}^{3}$, close to the standard structure, is represented by a finite-dimensional subvariety of $T_{0}^{\prime}$.

\section{ACKNOWLEDGMENTS}

The second named author would like to thank MIT for their hospitality and Richard Melrose for explaining his results on operators with symplectic characteristics of codimension two, [Me], and the regularity theory for elliptic operators of type $\left(\frac{1}{2}, \frac{1}{2}\right)$. We would also like to thank the referee for finding an error in an earlier version of this paper and for his very useful report.

\section{REFERENCES}

[BG] Richard Beals and Peter Greiner, Calculus on Heisenberg manifolds, Ann. of Math. Stud., vol. 119, Princeton Univ. Press, New Jersey, 1988, pp. 1-194.

[BM] Louis Boutet de Monvel, Integration des equations de Cauchy-Riemann induites formelles, Seminar Goulaouic-Lions-Schwartz (1974/75), IX.1-IX.13.

[CL] J. H. Cheng and John M. Lee, The Burns-Epstein invariant and deformations of CR-structures (to appear).

[E] Y. Eliashberg, Classification of overtwisted contact structures on 3-manifolds, Invent. Math. 98 (1989), 623-638.

[GK] Robert E. Greene and Steven G. Krantz, Stability of the caratheodory and Kobayashi metrics and applications to biholomorphic mappings, Proc. Sympos. Pure Math., vol. 41, Amer. Math. Soc., Providence, RI, 1984, 77-93.

[Ka] Tosio Kato, Perturbation theory for linear operators, 2nd ed., Springer-Verlag, Berlin, Heidelberg, and New York, 1980. 
[Ko] J. J. Kohn, The range of the tangential Cauchy-Riemann operator, Duke Math. J. 53 (1986), 525-545.

[L] John M. Lee, private communication, January 1990.

[M] Richard B. Melrose, The wave equation for a hypoelliptic operator with symplectic characteristics of codimension two, J. D'Analyse Math. 44 (1984/85), 134-182.

[N] L. Nirenberg, On a problem of Hans Lewy, Uspekhi Math. Nauk 29 (1974), 241-251. (Russian).

[R] Jean-Pierre Rosay, Sur une charactérisation de la boule parmi les domaines de $\mathbb{C}^{n}$ par son groupe d'automorphisms, Ann. Inst. Fourier (Grenoble) 24 (1979), 91-97.

[Ro] Hugo Rossi, Attaching analytic spaces to an analytic space along a pseudoconvex boundary, Proc. Conf. on Complex Manifolds (A. Aeppli, E. Calabi, R. Röhrl, eds.), Springer-Verlag, Berlin, Heidelberg, and New York, 1965.

Department of Mathematics, University of Michigan, Ann Arbor, Michigan 48109

Department of Mathematics, University of Pennsylvania, Philadelphia, PennsylVANIA 19104 